\title{
AN ADAPTIVE FINITE ELEMENT METHOD FOR THE EDDY CURRENT MODEL WITH CIRCUIT/FIELD COUPLINGS
}

\author{
JUNQING CHEN*, ZHIMING $\mathrm{CHEN}^{\dagger}$, TAO CUI ${ }^{\ddagger}$, AND LIN-BO ZHANG ${ }^{\S}$
}

\begin{abstract}
We develop an adaptive finite element method for solving the eddy current model with voltage excitations for complicated three dimensional structures. The mathematical model is based on the $\mathbf{A}-\phi$ formulation whose well-posedness is established. We derive the a posteriori error estimate for the finite element approximation of the model whose solution is not unique in the nonconducting region. Numerical experiments are provided which illustrate the competitive behavior of the proposed method.
\end{abstract}

Key words. Eddy current, circuit/field coupling, adaptivity, a posteriori error analysis, PHG package.

AMS subject classifications. 65N30, 65N55

1. Introduction. There are tremendous interests in practical applications to develop efficient electromagnetic analysis tools that are capable of wide-band analysis of very complicated geometries of conductor, see e.g. Zhu et al [33], Kamon et al [19]. One example is the analysis of interconnects where accurate estimates of the coupling impedances of complicated three dimensional structures are important for determining final circuit speeds or functionality. The standard problem in this case consists of the determination of the equivalent parameters in the domains where the full Maxwell equations or the magneto-quasi-static problem must be solved (Rubinacci et al [29]). There are great efforts in the engineering literature to solve the problem based on the volume integral method, see e.g. Ruehli [30], Heeb and Ruehli [14], [33], and [19].

In this paper we develop an adaptive finite element method for solving the magneto-quasi-static or eddy current model with voltage excitations for complicated three dimensional structures. The eddy current model with voltage or current excitations draws considerable attention in the literature, see e.g. Dular [13], Kettunen [17], [29], Bermudez et al [5], Hiptmair and Sterz [15]. The difficulty is the coupling of the global quantities such as the voltage and current with local quantities like electric and magnetic fields. Our approach in this paper to couple the local and global quantities is based on the $\mathbf{A}-\phi$ model in [29] where an integral formulation of the model is developed.

Let $\Omega$ be a simply connected bounded domain with a connected Lipschitz boundary $\Gamma$ which contains the conducting region $\Omega_{\mathrm{c}}$ and the nonconducting region $\Omega_{\mathrm{nc}}=$ $\Omega \backslash \bar{\Omega}_{\mathrm{c}}$. The conducting body $\Omega_{\mathrm{c}}$ is fed by $N$ external sinusoidal voltage generators

\footnotetext{
*Department of Mathematical Sciences, Tsinghua University, Beijing 100084, P.R. China. (jqchen@math.tsinghua.edu.cn)

$\dagger$ Institute of Computational Mathematics, Academy of Mathematics and Systems Science, Chinese Academy of Sciences, Beijing 100190, P.R. China. The work of this author was partially supported by China NSF under the grant 10428105 and by the National Basic Research Project under the grant 2005CB321701. (zmchen@lsec.cc.ac.cn)

${ }^{\ddagger}$ Institute of Computational Mathematics, Academy of Mathematics and Systems Science, Chinese Academy of Sciences, Beijing 100190, P.R. China. (tcui@lsec.cc.ac.cn)

$\S$ Institute of Computational Mathematics, Academy of Mathematics and Systems Science, Chinese Academy of Sciences, Beijing 100190, P.R. China. The work of this author was partially supported by the National Basic Research Project under the grant 2005 CB321702 and by China NSF under the grant 10531080. (zlb@lsec.cc.ac.cn).
} 


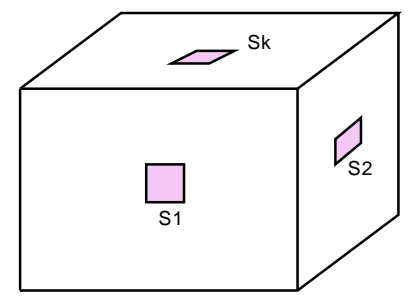

FIG. 1.1. The domain $\Omega$ and the electrodes $S_{j}, j=1, \cdots, N$.

through electrodes $S_{1}, \cdots, S_{N}$ which are connected subsets of $\Gamma$. The mathematical model consists of the standard eddy current equations in the frequency domain

$$
\begin{aligned}
& \nabla \times \mathbf{E}=-\mathbf{i} \omega \mathbf{B}, \\
& \nabla \times \mathbf{H}=\mathbf{J}+\mathbf{J}_{s},
\end{aligned}
$$

where $\mathbf{E}$ is the electric field, $\mathbf{H}$ is the magnetic field, $\mathbf{J}$ is the current density, $\mathbf{J}_{s}$ is the applied current density satisfying $\operatorname{div} \mathbf{J}_{s}=0$ in $\Omega$, and $\mathbf{B}$ is the magnetic flux density. We assume $\mathbf{B}=\mu \mathbf{H}$ and $\mathbf{J}=\sigma \mathbf{E}$ with $\mu>0$ the magnetic permeability and $\sigma$ the electric conductivity which is zero in $\Omega_{\mathrm{nc}}$ and constant in $\Omega_{\mathrm{c}}$. We assume the frequency $\omega$ and the magnetic permeability $\mu$ are positive constants in $\Omega$. We remark that the results of this paper can be extended to the case when $\mu$ is variable in space and possibly has small jumps.

We impose the following boundary conditions [13]

$$
\left.(\nabla \times \mathbf{E}) \cdot \mathbf{n}\right|_{\Gamma}=0, \quad \mathbf{E} \times\left.\mathbf{n}\right|_{\Gamma_{e}}=0,
$$

where $\Gamma=\partial \Omega$ is the boundary of the domain $\Omega, \Gamma_{e}=\cup_{j=1}^{N} S_{j}$ is the part of the boundary where the current is fed, and $\mathbf{n}$ is the unit outer normal to $\Gamma$. The first boundary condition ensures that there is no magnetic coupling between $\Omega$ and its exterior. By applying Theorem 3.6 in Girault and Raviart [3] to $\mathbf{v}=\nabla \times \mathbf{E}$, we know that $\mathbf{E}=\mathbf{\Phi}-\nabla U$ for some $\boldsymbol{\Phi} \in H_{0}(\operatorname{curl} ; \Omega)$ and $U \in H^{1}(\Omega)$. Thus $\mathbf{E} \times \mathbf{n}=-\nabla U \times \mathbf{n}$ for some boundary potential $U \in H^{1 / 2}(\Gamma)$. The second boundary condition in (1.3) then implies that $U=U_{j}$ on $S_{j}$ for some constant $U_{j}, j=1, \cdots, N$. Moreover, the existence of the tangential potential $U$ implies that $\mathbf{E}$ is a conservative field on the boundary, i.e. $\int_{\gamma} \mathbf{E} \cdot d \mathbf{l}=0$ for any closed path $\gamma$ on the boundary. This justifies that $U_{j}$ is in fact the voltage.

Based on this observation, the eddy current model (1.1)-(1.2) with the boundary condition (1.3) can be transformed to the following $\mathbf{A}-\phi$ form (see Section 2)

$$
\begin{aligned}
\nabla \times \nabla \times \mathbf{A}+\mathbf{i} \omega \sigma \mu \mathbf{A} & =-\sigma \mu \nabla \phi_{0}+\mu \mathbf{J}_{s} \quad \text { in } \Omega, \\
\mathbf{A} \times \mathbf{n} & =0 \quad \text { on } \Gamma .
\end{aligned}
$$

Here $\mathbf{A}$ is the magnetic vector potential and $\phi_{0} \in H^{1}(\Omega)$ is any function that satisfies $\phi_{0}=U_{j}$ on $S_{j}, j=1, \cdots, N$. It can be shown that the quantities of physical interests like the electric fields or the current densities depend only on the values $U_{j}$ and are independent of the particular form of $\phi_{0}$.

In the practical applications, the conducting body $\Omega_{\mathrm{c}}$ consists of complicated multiply-connected conductors with sharp edges and corners which implies that the magnetic potential A may have very strong singularities. Moreover, the well-known 
skin effect and the proximity effect make the standard finite element method with uniform mesh refinements inefficient. We recall that the skin effect refers to the current flows close to the boundary of the conductors and the proximity effect refers to the current flows on the adjacent boundary in each conductor if two conductors are put close to each other. In this paper we propose to solve (1.4)-(1.5) by the adaptive finite element method based on a posteriori error estimates.

A posteriori error estimates are computable quantities in terms of the discrete solution and known data that measure the actual discrete errors without the knowledge of exact solutions. They are essential in designing algorithms for mesh modification which equi-distribute the computational effort and optimize the computation. A posteriori error estimate and adaptive edge element method have been recently studied for the Maxwell cavity problem in Chen et al [12], for the time domain eddy current problem in Zheng et al [32], and for the electromagnetic scattering problems in Chen and Chen [10]. The extensive numerical experiments in [12] indicate that the adaptive methods based on the a posteriori error estimates have the very desirable quasi-optimality property: the energy error decays like $N^{-1 / 3}$ for the Nédéléc lowest order edge element, where $N$ is the number of degrees of freedom. We also refer to Beck et al [4] and Monk [23] for the earlier work on the a posteriori error estimates for the Maxwell equations.

The solution of (1.4)-(1.5) is not unique in the nonconducting region $\Omega_{\mathrm{nc}}$. This difficulty can be treated by introducing the Coulomb gauge $\operatorname{div} \mathbf{A}=0$ in $\Omega_{\mathrm{nc}}$ and formulate the problem as the saddle point form (see e.g. Chen et al [11] and Reitzinger and Schoberl [28]). In [28] an AMG algorithm is developed to solve the regularized curl-curl problem motivated by the saddle point formulation. Our approach here is motivated by the recent studies in the preconditioning of the Maxwell equation by Hiptmair and $\mathrm{Xu}[16]$ and its implementation in the software package hypre [18]. We will solve the problem (1.4)-(1.5) which has non-unique solutions directly by the adaptive edge element method based on the a posteriori error estimate for approximating non-unique solutions.

The layout of the paper is as follows. In Section 2 we introduce the mathematical model to be solved in this paper and prove its well-posedness. In Section 3 we introduce the finite element approximation and derive the a posteriori error estimate. In Section 4 we report our extensive numerical experiments based on the parallel adaptive finite element package PHG $[26,27]$ and the implementation of the Hiptmair and $\mathrm{Xu}$ preconditioner in Kolev and Vassilevski [20], [21] for systems with non-unique solutions.

2. The mathematical model. Let $\Omega$ be a simply connected bounded domain with a connected Lipschitz boundary $\Gamma$ which contains the conducting region $\Omega_{\mathrm{c}}$ and nonconducting region $\Omega_{\mathrm{nc}}=\Omega \backslash \bar{\Omega}_{\mathrm{c}}$. The conducting body $\Omega_{\mathrm{c}}$ is fed by $N$ external sinusoidal voltage generators through electrodes $S_{1}, \cdots, S_{N}$ which are connected subsets of $\Gamma$. We also allow $\Omega_{\mathrm{c}}$ having connected conductors $D_{i}, i=1, \cdots, I$, which lie inside $\Omega$ and are not connected with the electrodes. By the boundary condition (1.3), we know that there is a boundary potential $U \in H^{1 / 2}(\Gamma)$ such that $\mathbf{E} \times \mathbf{n}=-\nabla U \times \mathbf{n}$. Let $\varphi \in H^{1}(\Omega)$ such that $\varphi=U$ on $\Gamma$ and define $\mathbf{A}^{\prime}$ such that $\mathbf{E}=-\mathbf{i} \omega \mathbf{A}^{\prime}-\nabla \varphi$. Then $\mathbf{A}^{\prime}$ satisfies the boundary condition

$$
\mathbf{A}^{\prime} \times \mathbf{n}=0 \quad \text { on } \Gamma .
$$

Moreover, we deduce from (1.1)-(1.2) that

$$
\nabla \times \nabla \times \mathbf{A}^{\prime}+\sigma \mu\left(\mathbf{i} \omega \mathbf{A}^{\prime}+\nabla \varphi\right)=\mu \mathbf{J}_{s} \quad \text { in } \Omega .
$$


Let $\phi_{0} \in H^{1}(\Omega)$ be any function that satisfies $\phi_{0}=U_{j}$ on $S_{j}, j=1, \cdots, N$, and define $\varphi^{\prime}=\varphi-\phi_{0}$. Then $\varphi^{\prime} \in H^{1}(\Omega)$ and $\varphi^{\prime}=0$ on $S_{j}, j=1, \cdots, N$. Now let $\tilde{\varphi}^{\prime}$ be the extension of $\left.\varphi^{\prime}\right|_{\Omega_{\mathrm{c}}}$ to $\Omega_{\mathrm{nc}}$ such that $\tilde{\varphi}^{\prime}=0$ on $\Gamma$. Then since $\sigma=0$ in $\Omega_{\mathrm{nc}}$, we have from (2.2) that

$$
\nabla \times \nabla \times \mathbf{A}^{\prime}+\sigma \mu\left(\mathbf{i} \omega \mathbf{A}^{\prime}+\nabla \tilde{\varphi}^{\prime}\right)+\sigma \mu \nabla \phi_{0}=\mu \mathbf{J}_{s} \quad \text { in } \Omega .
$$

If we define $\mathbf{A}=\mathbf{A}^{\prime}+\frac{1}{\mathrm{i} \omega} \nabla \tilde{\varphi}^{\prime}$, then by (2.1) and $\tilde{\varphi}^{\prime}=0$ on $\Gamma$, we know that $\mathbf{A}$ satisfies the boundary condition

$$
\mathbf{A} \times \mathbf{n}=0 \quad \text { on } \Gamma .
$$

Moreover, from (2.3), A satisfies

$$
\nabla \times \nabla \times \mathbf{A}+\mathbf{i} \sigma \mu \omega \mathbf{A}=-\sigma \mu \nabla \phi_{0}+\mu \mathbf{J}_{s} \quad \text { in } \Omega .
$$

For practical implementation, the computational domain $\Omega$ should be dimensionless. Let $s>0$ be the characteristic size of the domain and set $\mathbf{x}^{\prime}=\mathbf{x} / s$, we obtain the dimensionless version of the above equation (here we still denote $\Omega$ the non-dimenionalized domain)

$$
\nabla \times \nabla \times \mathbf{A}+\mathbf{i} s^{2} \sigma \mu \omega \mathbf{A}=-s \sigma \mu \nabla \phi_{0}+s^{2} \mu \mathbf{J}_{s} \quad \text { in } \Omega .
$$

In practical applications, the eddy current $\mathbf{J}=\sigma \mathbf{E}$ is of particular interest. The following lemma shows that the eddy current is independent of the particular form of $\phi_{0}$.

Lemma 2.1. For any fixed $\phi_{0} \in H^{1}(\Omega)$, the solution $\mathbf{A}$ of (2.4)-(2.5) is unique in $\Omega_{\mathrm{c}}$. Moreover, the eddy current $\mathbf{J}=\sigma \mathbf{E}=\sigma\left(-\mathbf{i} \omega \mathbf{A}-s^{-1} \nabla \phi_{0}\right)$ depends only on the voltage $U_{j}$ on the electrodes $S_{j}, j=1, \cdots, N$, and is independent of the particular form of the function $\phi_{0} \in H^{1}(\Omega)$ such that $\phi_{0}=U_{j}$ on $S_{j}, j=1, \cdots, N$.

The proof of the lemma is obvious and we omit the details. To study the wellposedness of the problem (2.4)-(2.5), we elaborate more on the assumptions on the geometry of the conducting region $\Omega_{\mathrm{c}}$. Let $\Gamma_{\mathrm{c}}=\partial \Omega_{\mathrm{c}}$ and $\Gamma_{\mathrm{nc}}=\partial \Omega_{\mathrm{nc}}$. We know that $\Gamma_{\mathrm{c}} \cap \Gamma=\cup_{j=1}^{N} S_{j}$, where $S_{j}, j=1, \cdots, N$, are the electrodes where the outside voltage is fed. Let $\Gamma_{i}=\partial D_{i}, i=1, \cdots, I$, be the boundary of isolated conductors $D_{i}$ that lie inside $\Omega$. Then the common boundary of the conducting and nonconducting region $\Gamma_{\mathrm{c}} \cap \Gamma_{\mathrm{nc}}=\Gamma_{0} \cup\left(\cup_{i=1}^{I} \Gamma_{i}\right)$, where $\Gamma_{0}$ is part of the boundary that is connected with the external boundary $\Gamma$. It is obvious that the solution of (2.4)-(2.5) is not unique. For, if $\mathbf{A}$ is some solution of (2.4)-(2.5) and $\psi \in H^{1}\left(\Omega_{\mathrm{nc}}\right)$ such that $\psi=0$ on $\Gamma_{0} \cup\left(\Gamma_{\mathrm{nc}} \cap \Gamma\right)$ and $\psi=\psi_{i}$ for some constant $\psi_{i}$ on $\Gamma_{i}, i=1, \cdots, I$, then

$$
\tilde{\mathbf{A}}= \begin{cases}\mathbf{A}+\nabla \psi & \text { in } \Omega_{\mathrm{nc}} \\ \mathbf{A} & \text { in } \Omega_{\mathrm{c}}\end{cases}
$$

is also a solution of (2.4)-(2.5).

Let $H_{S}^{1}\left(\Omega_{\mathrm{nc}}\right)=\left\{v \in H^{1}\left(\Omega_{\mathrm{nc}}\right): v=0\right.$ on $\Gamma_{0} \cup\left(\Gamma_{\mathrm{nc}} \cap \Gamma\right), v=$ const on $\Gamma_{i}, i=$ $1, \cdots, I\}$. We seek the solution $\mathbf{A}$ in the following subspace of $H_{0}(\mathbf{c u r l} ; \Omega)$

$$
\mathbf{X}=\left\{\mathbf{G} \in H_{0}(\mathbf{c u r l} ; \Omega):(\mathbf{G}, \nabla v)_{\Omega_{\mathrm{nc}}}=0, \quad \forall v \in H_{S}^{1}\left(\Omega_{\mathrm{nc}}\right)\right\} .
$$

Here $(\cdot, \cdot)_{D}$ stands for the inner product of $L^{2}(D)$ and the subscript $D$ is omitted when $D=\Omega$. It is clear that $\mathbf{G} \in \mathbf{X}$ is equivalent to impose the following gauge conditions

$$
\operatorname{div} \mathbf{G}=0 \quad \text { in } \Omega_{\mathrm{nc}}, \quad\langle\mathbf{G} \cdot \mathbf{n}, 1\rangle_{\Gamma_{i}}=0, \quad i=1, \cdots, I,
$$


where $\langle\cdot, \cdot\rangle_{\Gamma_{i}}$ is the duality pairing between $H^{-1 / 2}\left(\Gamma_{i}\right)$ and $H^{1 / 2}\left(\Gamma_{i}\right)$.

Define the sesquilinear form $a(\cdot, \cdot): H_{0}(\operatorname{curl} ; \Omega) \times H_{0}(\operatorname{curl} ; \Omega) \rightarrow \mathbb{C}$

$$
a(\mathbf{A}, \mathbf{G})=(\nabla \times \mathbf{A}, \nabla \times \mathbf{G})+\mathbf{i} s^{2} \omega \mu(\sigma \mathbf{A}, \mathbf{G})_{\Omega_{\mathrm{c}}} .
$$

The weak formulation of the problem (2.4)-(2.5) with the gauge (2.6) then reads as follows: Find $\mathbf{A} \in \mathbf{X}$ such that

$$
a(\mathbf{A}, \mathbf{G})=-s \mu\left(\sigma \nabla \phi_{0}, \mathbf{G}\right)_{\Omega_{\mathrm{c}}}+s^{2} \mu\left(\mathbf{J}_{s}, \mathbf{G}\right), \quad \forall \mathbf{G} \in \mathbf{X} .
$$

To proceed, we recall some notation. For any bounded domain $D \subset \mathbb{R}^{3}$ with a Lipschitz boundary $\Gamma_{D}$ and the unit outer normal $\mathbf{n}_{D}$ to $\Gamma_{D}$, the trace of functions in $H(\mathbf{c u r l} ; D)$ belongs to

$$
H^{-1 / 2}\left(\operatorname{Div}_{\Gamma_{\mathrm{D}}} ; \Gamma_{\mathrm{D}}\right)=\left\{\boldsymbol{\lambda} \in \mathrm{V}_{\pi}\left(\Gamma_{\mathrm{nc}}\right)^{\prime}: \operatorname{div}_{\Gamma_{\mathrm{D}}} \boldsymbol{\lambda} \in \mathrm{H}^{-1 / 2}\left(\Gamma_{\mathrm{D}}\right)\right\} .
$$

Here $V_{\pi}\left(\Gamma_{D}\right)^{\prime}$ is the conjugate space of $V_{\pi}\left(\Gamma_{D}\right)=\pi\left(H^{1 / 2}\left(\Gamma_{D}\right)^{3}\right)$, where for any $\mathbf{v} \in$ $H^{1 / 2}\left(\Gamma_{D}\right)^{3}, \pi(\mathbf{v})=\mathbf{n}_{D} \times \mathbf{v} \times \mathbf{n}_{D}$, and $\operatorname{div}_{\Gamma_{D}} \boldsymbol{\lambda}$ is the surface divergence of $\boldsymbol{\lambda}$ on $\Gamma_{D}$. $H^{-1 / 2}\left(\operatorname{Div}_{\Gamma_{\mathrm{D}}} ; \Gamma_{\mathrm{D}}\right)$ is a Hilbert space under the graph norm. It is shown in Buffa et al [8] that the tangential trace operator $\gamma: H\left(\operatorname{curl} ; \Omega_{D}\right) \rightarrow H^{-1 / 2}\left(\operatorname{Div}_{\Gamma_{\mathrm{D}}} ; \Gamma_{\mathrm{D}}\right)$, $\gamma(\mathbf{v})=\mathbf{v} \times \mathbf{n}_{D}$, is surjective. In this paper we will use the following equivalent norm of $H^{-1 / 2}\left(\operatorname{Div}_{\Gamma_{\mathrm{D}}} ; \Gamma_{\mathrm{D}}\right)$

$$
\|\boldsymbol{\lambda}\|_{H^{-1 / 2}\left(\operatorname{Div}_{\Gamma_{\mathrm{D}}} ; \Gamma_{\mathrm{D}}\right)}=\inf \left\{\|\mathbf{G}\|_{H(\operatorname{curl} ; D)}: \mathbf{G} \in H(\operatorname{curl} ; D), \mathbf{G} \times \mathbf{n}_{D}=\boldsymbol{\lambda} \text { on } \Gamma_{D}\right\} .
$$

Lemma 2.2. The problem (2.7) has a unique solution $\mathbf{A} \in \mathbf{X}$.

Proof. For any $\mathbf{G} \in \mathbf{X}$, its trace $\mathbf{G} \times \mathbf{n}$ on $\Gamma_{\mathrm{nc}}$ belongs to $H^{-1 / 2}\left(\operatorname{Div}_{\Gamma_{\mathrm{nc}}} ; \Gamma_{\mathrm{nc}}\right)$. Let $\mathbf{B} \in H\left(\mathbf{c u r l} ; \Omega_{\mathrm{nc}}\right)$ satisfy $\mathbf{B} \times \mathbf{n}=\mathbf{G} \times \mathbf{n}$ on $\Gamma_{\mathrm{nc}}$ and

$$
(\nabla \times \mathbf{B}, \nabla \times \mathbf{v})_{\Omega_{\mathrm{nc}}}+(\mathbf{B}, \mathbf{v})_{\Omega_{\mathrm{nc}}}=0, \quad \forall \mathbf{v} \in H_{0}\left(\mathbf{c u r l} ; \Omega_{\mathrm{nc}}\right) .
$$

By the Lax-Milgram lemma, $(2.8)$ has a unique solution $\mathbf{B} \in H\left(\mathbf{c u r l} ; \Omega_{\mathrm{nc}}\right)$. It is obvious that $\|\mathbf{B}\|_{H\left(\operatorname{curl} ; \Omega_{\mathrm{nc}}\right)} \leq\|\mathbf{G} \times \mathbf{n}\|_{H^{-1 / 2}\left(\operatorname{Div}_{\Gamma_{\mathbf{n c}}} ; \Gamma_{\mathrm{nc}}\right)}$. Since $\mathbf{G} \times \mathbf{n}=0$ on $\Gamma$,

$$
\|\mathbf{G} \times \mathbf{n}\|_{H^{-1 / 2}\left(\operatorname{Div}_{\Gamma_{\mathrm{nc}}} ; \Gamma_{\mathrm{nc}}\right)}=\|\mathbf{G} \times \mathbf{n}\|_{H^{-1 / 2}\left(\operatorname{Div}_{\Gamma_{\mathrm{C}}} ; \Gamma_{\mathrm{C}}\right)} .
$$

Thus $\|\mathbf{B}\|_{H\left(\mathbf{c u r l} ; \Omega_{\mathrm{nc}}\right)} \leq\|\mathbf{G}\|_{H\left(\mathbf{c u r l} ; \Omega_{\mathrm{c}}\right)}$.

Since $\mathbf{G} \in \mathbf{X}$, we know that $\mathbf{G}$ satisfies (2.6). For any $\phi \in H_{S}^{1}\left(\Omega_{\mathrm{nc}}\right)$, we have $\nabla \phi \times \mathbf{n}=0$ on $\Gamma_{\mathrm{nc}}$. By taking $\nabla \phi$ in (2.8), it is easy to see that $\mathbf{B} \in \mathbf{X}$. Thus $\mathbf{B}$ also satisfies (2.6). Furthermore, $(\mathbf{G}-\mathbf{B}) \times \mathbf{n}=0$ on $\Gamma_{\text {nc }}$. By the embedding theorem in Amrouche et al [1] we know that

$$
\|\mathbf{G}-\mathbf{B}\|_{L^{2}\left(\Omega_{\mathrm{nc}}\right)} \leq C\|\nabla \times(\mathbf{G}-\mathbf{B})\|_{L^{2}\left(\Omega_{\mathrm{nc}}\right)} .
$$

Therefore

$$
\begin{aligned}
\|\mathbf{G}\|_{L^{2}\left(\Omega_{\mathrm{nc}}\right)} & \leq C\|\nabla \times \mathbf{G}\|_{L^{2}\left(\Omega_{\mathrm{nc}}\right)}+C\|\mathbf{B}\|_{H\left(\operatorname{curl} ; \Omega_{\mathrm{nc}}\right)} \\
& \leq C\|\nabla \times \mathbf{G}\|_{L^{2}\left(\Omega_{\mathrm{nc}}\right)}+C\|\mathbf{G}\|_{H\left(\operatorname{curl} ; \Omega_{\mathrm{c}}\right)} \\
& \leq C\|\nabla \times \mathbf{G}\|_{L^{2}(\Omega)}+C\|\mathbf{G}\|_{L^{2}\left(\Omega_{\mathrm{c}}\right)} .
\end{aligned}
$$

This yields

$$
|a(\mathbf{G}, \mathbf{G})| \geq C \min \left(1, s^{2} \omega \sigma \mu\right)\|\mathbf{G}\|_{H(\mathbf{c u r l} ; \Omega)}^{2}, \quad \forall \mathbf{G} \in \mathbf{X} .
$$

The lemma now follows from the Lax-Milgram lemma. $\mathrm{C}$

We remark that the constant $C$ in (2.10) depends on the size of the domain $\Omega_{\mathrm{nc}}$ through the embedding constant in (2.9). 
3. The finite element method and a posteriori error analysis. In this section we introduce the finite element method for solving (2.4)-(2.5). Let $\mathcal{M}_{h}$ be a regular tetrahedral triangulation of $\Omega$ and $\mathcal{F}_{h}$ be the set of faces not lying on $\Gamma$. We assume $\Omega_{\mathrm{c}}$ is polyhedral so that the elements in $\mathcal{M}_{h}$ are contained either in $\Omega_{\mathrm{c}}$ or in $\Omega_{\mathrm{nc}}$.

The Nédélec lowest order edge element space $\mathbf{U}_{h}$ over $\mathcal{M}_{h}$ is defined by [24]

$$
\begin{aligned}
\mathbf{U}_{h}:= & \left\{\mathbf{u} \in H(\mathbf{c u r l} ; \Omega): \mathbf{u} \times\left.\mathbf{n}\right|_{\Gamma}=\mathbf{0}\right. \text { and } \\
& \left.\left.\mathbf{u}\right|_{T}=\mathbf{a}_{T}+\mathbf{b}_{T} \times \mathbf{x} \quad \text { with } \quad \mathbf{a}_{T}, \mathbf{b}_{T} \in \mathbb{R}^{3}, \quad \forall T \in \mathcal{M}_{h}\right\} .
\end{aligned}
$$

Degrees of freedom on every $T \in \mathcal{M}_{h}$ are $\int_{E_{i}} \mathbf{u} \cdot d \mathbf{l}, \quad i=1, \cdots, 6$, where $E_{1}, \cdots, E_{6}$ are the six edges of $T$. For any $T \in \mathcal{M}_{h}$ and $F \in \mathcal{F}_{h}$, we denote the diameters of $T$ and $F$ by $h_{T}$ and $h_{F}$ respectively.

The finite element approximation to (2.4)-(2.5) is: Find $\mathbf{A}_{h} \in \mathbf{U}_{h}$ such that

$$
a\left(\mathbf{A}_{h}, \mathbf{G}_{h}\right)=-s \mu\left(\sigma \nabla \phi_{0}, \mathbf{G}_{h}\right)_{\Omega_{\mathrm{c}}}+s^{2} \mu\left(\mathbf{J}_{s}, \mathbf{G}_{h}\right), \quad \forall \mathbf{G}_{h} \in \mathbf{U}_{h}
$$

The solution of the problem (3.1) is not unique which introduces difficulty in deriving the a posteriori error estimate. To overcome the difficulty, we introduce the finite element approximation of the gauged problem (2.7). Let $\mathbf{X}_{h}$ be the subspace of $\mathbf{U}_{h}$ with the discrete gauge condition

$$
\mathbf{X}_{h}=\left\{\mathbf{G}_{h} \in \mathbf{U}_{h}:\left(\mathbf{G}_{h}, \nabla v_{h}\right)_{\Omega_{\mathrm{nc}}}=0, \quad \forall v_{h} \in V_{h}\left(\Omega_{\mathrm{nc}}\right)\right\},
$$

where $V_{h}\left(\Omega_{\mathrm{nc}}\right) \subset H_{S}^{1}\left(\Omega_{\mathrm{nc}}\right)$ is the conforming linear finite element space over the mesh in $\Omega_{\mathrm{nc}}$. We remark that $\mathbf{X}_{h} \not \subset \mathbf{X}$.

The finite element approximation of the gauged problem (2.7) is: Find $\hat{\mathbf{A}}_{h} \in \mathbf{X}_{h}$ such that

$$
a\left(\hat{\mathbf{A}}_{h}, \mathbf{G}_{h}\right)=-s \mu\left(\sigma \nabla \phi_{0}, \mathbf{G}_{h}\right)_{\Omega_{\mathrm{c}}}+s^{2} \mu\left(\mathbf{J}_{s}, \mathbf{G}_{h}\right), \quad \forall \mathbf{G}_{h} \in \mathbf{X}_{h} .
$$

LEMma 3.1. The problem (3.2) has a unique solution $\hat{\mathbf{A}}_{h} \in \mathbf{X}_{h}$.

Proof. The proof is standard and we include here for the sake of completeness. It is clear that we only need to prove the uniqueness for which we may let $\phi_{0}=0$ and $\mathbf{J}_{s}=0$ in (3.2). Thus $\nabla \times \hat{\mathbf{A}}_{h}=0$ in $\Omega$ and $\hat{\mathbf{A}}_{h}=0$ in $\Omega_{\mathrm{c}}$. Since $\Omega$ is simply connected we know that $\hat{\mathbf{A}}_{h}=\nabla \psi_{h}$ for some conforming linear finite element function $\psi_{h}$ such that $\psi_{h}=0$ on $\Gamma$. $\hat{\mathbf{A}}_{h}=0$ in $\Omega_{\mathrm{c}}$ implies that $\psi_{h}$ is constant on each connected components of $\Omega_{\mathrm{c}}$. Consequently, $\psi_{h} \in V_{h}\left(\Omega_{\mathrm{nc}}\right)$. Thus by the discrete gauge condition $\nabla \psi_{h}=0$ in $\Omega_{\mathrm{nc}}$. This completes the proof. $\mathrm{c}$

As a consequence of Lemma 3.1 we know that for the problem (3.1) there exists a solution $\mathbf{A}_{h}$. For any solution $\mathbf{A}_{h} \in \mathbf{U}_{h}$ of the problem (3.1), let $\phi_{h} \in V_{h}\left(\Omega_{\mathrm{nc}}\right)$ satisfy

$$
\left(\nabla \phi_{h}, \nabla v_{h}\right)_{\Omega_{\mathrm{nc}}}=\left(\mathbf{A}_{h}, \nabla v_{h}\right)_{\Omega_{\mathrm{nc}}}, \quad \forall v_{h} \in V_{h}\left(\Omega_{\mathrm{nc}}\right)
$$

We extend $\phi_{h}$ as a finite element function to the whole domain $\Omega$ by setting the value of $\phi_{h}$ as constant in each of the connected components of $\Omega_{\mathrm{c}}$ with the value the same as its value on the boundary of the connected component.

Lemma 3.2. We have $\hat{\mathbf{A}}_{h}=\mathbf{A}_{h}-\nabla \phi_{h}$.

Proof. By (3.3) we know that $\mathbf{A}_{h}-\nabla \phi_{h} \in \mathbf{X}_{h}$. On the other hand, since $\nabla \phi_{h}=0$ in $\Omega_{\mathrm{c}}, a\left(\mathbf{A}_{h}-\nabla \phi_{h}, \mathbf{G}_{h}\right)=a\left(\mathbf{A}_{h}, \mathbf{G}_{h}\right)$ for any $\mathbf{G}_{h} \in \mathbf{U}_{h}$. The lemma follows from the uniqueness of the solution of the problem (3.2) in Lemma 3.1. 
To derive a posteriori error estimates, we require the Scott-Zhang interpolant $\mathcal{I}_{h}: H^{1}(\Omega) \rightarrow V_{h}(\Omega)[31]$ and the Beck-Hiptmair-Hoppe-Wohlmuth interpolant $\Pi_{h}:$ $\mathbf{H}^{1}(\Omega) \cap H(\mathbf{c u r l} ; \Omega) \rightarrow \mathbf{U}_{h}[4]$, where $V_{h}(\Omega)$ is the standard piecewise linear $H^{1}$ conforming finite element space over $\mathcal{M}_{h}$. It is known that $\mathcal{I}_{h}$ and $\Pi_{h}$ satisfy the following approximation and stability properties: for any $T \in \mathcal{M}_{h}, F \in \mathcal{F}_{h}, \phi_{h} \in$ $V_{h}(\Omega), \phi \in H^{1}(\Omega)$,

$$
\begin{aligned}
& \mathcal{I}_{h} \phi_{h}=\phi_{h}, \quad\left\|\nabla \mathcal{I}_{h} \phi\right\|_{0, T} \leq C|\phi|_{1, D_{T}}, \\
& \left\|\phi-\mathcal{I}_{h} \phi\right\|_{0, T} \leq C h_{T}|\phi|_{1, D_{T}}, \quad\left\|\phi-\mathcal{I}_{h} \phi\right\|_{0, F} \leq C h_{F}^{1 / 2}|\phi|_{1, D_{F}},
\end{aligned}
$$

and for any $T \in \mathcal{M}_{h}, F \in \mathcal{F}_{h}, \mathbf{w}_{h} \in \mathbf{U}_{h}, \mathbf{w} \in H(\operatorname{curl} ; \Omega)$,

$$
\begin{aligned}
& \Pi_{h} \mathbf{w}_{h}=\mathbf{w}_{h}, \quad\left\|\Pi_{h} \mathbf{w}\right\|_{H(\mathbf{c u r l} ; T)} \leq C\|\mathbf{w}\|_{1, D_{T}}, \\
& \left\|\mathbf{w}-\Pi_{h} \mathbf{w}\right\|_{0, T} \leq C h_{T}|\mathbf{w}|_{1, D_{T}}, \quad\left\|\mathbf{w}-\Pi_{h} \mathbf{w}\right\|_{0, F} \leq C h_{F}^{1 / 2}|\mathbf{w}|_{1, D_{F}},
\end{aligned}
$$

where $D_{A}$ is the union of elements in $\mathcal{M}_{h}$ with non-empty intersection with $A, A=T$ or $F$.

For any face $F \in \mathcal{F}_{h}$, assuming $F=T_{1} \cap T_{2}, T_{1}, T_{2} \in \mathcal{M}_{h}$ and the unit normal $\mathbf{n}$ points from $T_{2}$ to $T_{1}$, we denote the jump of a function $v$ across $F$ by $\llbracket v \rrbracket_{F}:=\left.v\right|_{T_{1}}-\left.v\right|_{T_{2}}$.

The following theorem is the main result of this section.

Theorem 3.3. Let $\mathbf{A}$ be the solution of (2.7) and $\mathbf{A}_{h}$ be the solution of (3.1). Let $\phi_{h}$ be defined according to (3.3). There exists a constant $C$ depending only on the minimum angle of the mesh $\mathcal{M}_{h}$ and the size of the domain $\Omega_{\mathrm{nc}}$ such that

$$
\left\|\nabla \times\left(\mathbf{A}-\mathbf{A}_{h}\right)\right\|_{L^{2}(\Omega)}+\alpha\left\|\mathbf{A}-\mathbf{A}_{h}\right\|_{L^{2}\left(\Omega_{\mathrm{c}}\right)} \leq C \min (1, \alpha)^{-1}\left(\sum_{T \in \mathcal{M}_{h}} \eta_{T}^{2}\right)^{1 / 2}
$$

where $\alpha=\sqrt{s^{2} \omega \sigma \mu}$ and, for any $T \in \mathcal{M}_{h}$,

$$
\begin{aligned}
\eta_{T}^{2} & =h_{T}^{2}\left\|s^{2} \mu \mathbf{J}_{s}-s^{2} \sigma \mu\left(s^{-1} \nabla \phi_{0}+\mathbf{i} \omega \mathbf{A}_{h}\right)\right\|_{L^{2}(T)}^{2} \\
& +h_{T}^{2}\left\|s^{2} \mu \sigma \operatorname{div}\left(s^{-1} \nabla \phi_{0}+\mathbf{i} \omega \mathbf{A}_{h}\right)\right\|_{L^{2}(T)}^{2} \\
& +\sum_{F \in \mathcal{F}, F \subset \partial T} h_{F}\left\|\llbracket \mathbf{n} \times \nabla \times \mathbf{A}_{h} \rrbracket_{F}\right\|_{L^{2}(F)}^{2} \\
& +\sum_{F \in \mathcal{F}, F \subset \partial T} h_{F}\left\|\llbracket s^{2} \sigma \mu\left(s^{-1} \nabla \phi_{0}+\mathbf{i} \omega \mathbf{A}_{h}\right) \cdot \mathbf{n} \rrbracket_{F}\right\|_{L^{2}(F)}^{2} .
\end{aligned}
$$

Proof. Let $\zeta \in H_{S}^{1}\left(\Omega_{\mathrm{nc}}\right)$ be the solution of the problem

$$
(\nabla \zeta, \nabla v)_{\Omega_{\mathrm{nc}}}=\left(\mathbf{A}_{h}, \nabla v\right)_{\Omega_{\mathrm{nc}}}, \quad \forall v \in H_{S}^{1}\left(\Omega_{\mathrm{nc}}\right) .
$$

The unique existence of $\zeta$ is guaranteed by the Lax-Milgram lemma. Since $\zeta \in$ $H_{S}^{1}\left(\Omega_{\mathrm{nc}}\right)$ we can extend $\zeta$ to the whole domain by letting $\zeta$ being constant on each of the connected components of $\Omega_{\mathrm{c}}$ whose value equals to its value on the boundary of the connected component. It is clear $\mathbf{A}_{h}-\nabla \zeta \in \mathbf{X}$.

Let $\mathbf{G}=\mathbf{A}-\mathbf{A}_{h}+\nabla \zeta$. Then $\mathbf{G} \in \mathbf{X}$. By (2.7)

$$
a(\mathbf{A}, \mathbf{G})=-s \mu\left(\sigma \nabla \phi_{0}, \mathbf{G}\right)_{\Omega_{\mathrm{c}}}+s^{2} \mu\left(\mathbf{J}_{s}, \mathbf{G}\right) .
$$


By the Birman-Solomyak decomposition [6], [12], we know that there exists a function $\mathbf{v} \in H^{1}(\Omega)^{3} \cap H_{0}(\mathbf{c u r l} ; \Omega)$ and a function $q \in H_{0}^{1}(\Omega)$ such that $\mathbf{G}=\mathbf{v}+\nabla q$ and

$$
\|\mathbf{v}\|_{H^{1}(\Omega)}+\|q\|_{H^{1}(\Omega)} \leq C\|\mathbf{G}\|_{H(\operatorname{curl} ; \Omega)} .
$$

Then $\mathbf{G}_{h}=\Pi_{h} \mathbf{v}+\nabla \mathcal{I}_{h} q \in \mathbf{U}_{h}$. Now, by (3.1)

$$
a\left(\mathbf{A}-\mathbf{A}_{h}, \mathbf{G}\right)=-s \mu\left(\sigma \nabla \phi_{0}, \mathbf{G}-\mathbf{G}_{h}\right)_{\Omega_{\mathrm{c}}}+s^{2} \mu\left(\mathbf{J}_{s}, \mathbf{G}-\mathbf{G}_{h}\right)-a\left(\mathbf{A}_{h}, \mathbf{G}-\mathbf{G}_{h}\right) .
$$

By using the condition $\operatorname{div} \mathbf{J}_{s}=0$ in $\Omega$ we can obtain

$$
\begin{aligned}
a\left(\mathbf{A}-\mathbf{A}_{h}, \mathbf{G}\right) & =\left(s^{2} \mu \mathbf{J}_{s}-s^{2} \mu \sigma\left(s^{-1} \nabla \phi_{0}+\mathbf{i} \omega \mathbf{A}_{h}\right), \mathbf{v}-\Pi_{h} \mathbf{v}\right) \\
& -\left(\nabla \times \mathbf{A}_{h}, \nabla \times\left(\mathbf{v}-\Pi_{h} \mathbf{v}\right)\right) \\
& -\left(s^{2} \sigma \mu\left(s^{-1} \nabla \phi_{0}+\mathbf{i} \omega \mathbf{A}_{h}\right), \nabla\left(q-\mathcal{I}_{h} q\right)\right),
\end{aligned}
$$

which, after integration by parts and using the standard argument in the finite element a posteriori error analysis, implies

$$
\left|a\left(\mathbf{A}-\mathbf{A}_{h}, \mathbf{G}\right)\right| \leq C\left(\sum_{T \in \mathcal{M}_{h}} \eta_{T}^{2}\right)^{1 / 2}\left(\|\mathbf{v}\|_{H^{1}(\Omega)}+\|q\|_{H^{1}(\Omega)}\right)
$$

By using (3.5) and (2.10) we deduce that

$$
\left|a\left(\mathbf{A}-\mathbf{A}_{h}, \mathbf{G}\right)\right| \leq C \min (1, \alpha)^{-1}\left(\sum_{T \in \mathcal{M}_{h}} \eta_{T}^{2}\right)^{1 / 2}|a(\mathbf{G}, \mathbf{G})|^{1 / 2} .
$$

Since $\zeta$ is constant in each of the connected components of $\Omega_{\mathrm{c}}, \nabla \zeta=0$ in $\Omega_{\mathrm{c}}$. Notice that $\mathbf{G}=\mathbf{A}-\mathbf{A}_{h}+\nabla \zeta$, we have $\left|a\left(\mathbf{A}-\mathbf{A}_{h}, \mathbf{G}\right)\right|=|a(\mathbf{G}, \mathbf{G})|$ and

$$
\begin{aligned}
\left|a\left(\mathbf{A}-\mathbf{A}_{h}, \mathbf{G}\right)\right| & =\left|a\left(\mathbf{A}-\mathbf{A}_{h}, \mathbf{A}-\mathbf{A}_{h}\right)\right| \\
& =\left\|\nabla \times\left(\mathbf{A}-\mathbf{A}_{h}\right)\right\|_{L^{2}(\Omega)}^{2}+\alpha^{2}\left\|\mathbf{A}-\mathbf{A}_{h}\right\|_{L^{2}\left(\Omega_{c}\right)}^{2} .
\end{aligned}
$$

This completes the proof. $\square$

Recall that $\phi_{0} \in H^{1}(\Omega)$ can be any function that satisfies $\phi_{0}=U_{j}$ on $S_{j}, j=$ $1, \cdots, N$. In this paper we choose $\phi_{0}$ as a piecewise linear function on the initial mesh so that $\phi_{0}$ is always a piecewise linear function in the subsequent adaptively refined meshes. With this choice we know that the second term in the estimator $\eta_{T}$ vanishes because for the lowest order Nédélec edge element, $\operatorname{div} \mathbf{A}_{h}=0$ in $T \in \mathcal{M}_{h}$. The following local lower bound of the a posteriori error estimator can be proved in a similar way as in [4].

TheOREM 3.4. There exists a constant $C$ depending only on the minimum angle of the mesh $\mathcal{M}_{h}$ such that for any $T \in \mathcal{M}_{h}$,

$$
\eta_{T} \leq C\left(\left\|\nabla \times\left(\mathbf{A}-\mathbf{A}_{h}\right)\right\|_{L^{2}(\tilde{T})}+\alpha^{2}\left\|\mathbf{A}-\mathbf{A}_{h}\right\|_{L^{2}\left(\Omega_{c} \cap \tilde{T}\right)}+\sum_{T \subset \tilde{T}} h_{T}\left\|\mathbf{f}-Q_{h} \mathbf{f}\right\|_{L^{2}(T)}\right)
$$

where $\tilde{T}$ is the union of $T$ and the adjacent elements of $T, \mathbf{f}=s^{2} \mu\left(\mathbf{J}_{s}-s^{-1} \sigma \nabla \phi_{0}\right)$, and $Q_{h}: L^{2}(T) \rightarrow P_{1}(T)$ is the $L^{2}$ projection to the space of linear functions on $T$. 
From Theorem 3.4 we know that

$$
\begin{aligned}
\left(\sum_{T \in \mathcal{M}_{h}} \eta_{T}^{2}\right)^{1 / 2} \leq & C \max (1, \alpha)\left(\left\|\nabla \times\left(\mathbf{A}-\mathbf{A}_{h}\right)\right\|_{L^{2}(\Omega)}+\alpha\left\|\mathbf{A}-\mathbf{A}_{h}\right\|_{L^{2}\left(\Omega_{c}\right)}\right) \\
& +C\left(\sum_{T \in \mathcal{M}_{h}} h_{T}^{2}\left\|\mathbf{f}-Q_{h} \mathbf{f}\right\|_{L^{2}(T)}^{2}\right)^{1 / 2}
\end{aligned}
$$

We notice that the upper bound constant in Theorem 3.3 is independent of $\alpha$ for $\alpha \geq 1$. On the other hand, the lower bound constant in (3.6) is independent of $\alpha$ for $\alpha \leq 1$. Therefore, our a posteriori error estimate is sharp up to a constant $\alpha$. In the case when $\alpha \leq 1$, one can absorb the quantity $\min (1, \alpha)^{-1}$ into the a posteriori error estimator so that the upper bound constant is independent of $\alpha$. For the numerical examples in the next section, the frequency ranges from 1 to $100 \mathrm{Ghz}$ and $s=10^{-6}$, the constant $\alpha$ ranges between 0.27 and 2.7 and so we keep the estimator in the form in Theorem 3.3.

4. Numerical examples. The implementation of the adaptive finite element method in this paper is based on the parallel adaptive finite element package PHG (Parallel Hierarchical Grid) [26, 27] which uses unstructured meshes and is based on MPI. The computations are performed on the cluster LSSC-II in the State Key Laboratory on Scientific and Engineering Computing of Chinese Academy of Sciences.

The adaptive algorithm is based on the a posteriori error estimate in Theorem 3.3. We define the global a posteriori error estimate and the maximal element error estimate over $\mathcal{T}_{h}$ respectively by

$$
\mathcal{E}:=\left(\sum_{T \in \mathcal{T}_{h}} \eta_{T}^{2}\right)^{1 / 2}, \quad \eta_{\max }=\max _{T \in \mathcal{T}_{h}} \eta_{T} .
$$

Now we describe the adaptive algorithm used in this paper.

Algorithm. Given a tolerance $T o l>0$ and the initial mesh $\mathcal{T}_{0}$. Set $\mathcal{T}_{h}=\mathcal{T}_{0}$.

1. Solve the discrete problem $(3.1)$ on $\mathcal{T}_{0}$.

2. Compute the local error estimator $\eta_{T}$ on each $T \in \mathcal{T}_{0}$, the global error estimate $\mathcal{E}$, and the maximal element error estimate $\eta_{\max }$.

3. While $\mathcal{E}>$ Tol do

- Refine the mesh $\mathcal{T}_{h}$ according to the following strategy

$$
\text { if } \eta_{T}>\frac{1}{2} \eta_{\max } \text {, refine the element } T \in \mathcal{T}_{h} \text {. }
$$

- Solve the discrete problem $(3.1)$ on $\mathcal{T}_{h}$.

- Compute the local error estimator $\eta_{T}$ on each $T \in \mathcal{T}_{h}$, the global error estimate $\mathcal{E}$, and the maximal element error estimate $\eta_{\max }$. end while.

The problem (3.1) which results in a singular algebraic system of equations can be solved by a preconditioned GMRES or MINRES method. The construction of the preconditioner follows from the method in [12] where a time-harmonic Maxwell cavity problem is considered. 

system

By splitting $\mathbf{A}_{h}=\operatorname{Re} \mathbf{A}_{h}+\mathbf{i} \operatorname{Im} \mathbf{A}_{h}$, the problem (3.1) results in an algebraic

$$
\left(\begin{array}{cc}
K & -M \\
-M & -K
\end{array}\right)\left(\begin{array}{l}
x \\
y
\end{array}\right)=\left(\begin{array}{l}
f \\
0
\end{array}\right)
$$

where $K_{i j}=\left(\nabla \times \boldsymbol{\Psi}_{i}, \nabla \times \boldsymbol{\Psi}_{j}\right), M_{i j}=\left(s^{2} \omega \mu \sigma \mathbf{\Psi}_{i}, \boldsymbol{\Psi}_{j}\right), f_{i}=\left(-s \sigma \mu \nabla \phi_{0}+s^{2} \mu \mathbf{J}_{s}, \boldsymbol{\Psi}_{i}\right)$, and $\boldsymbol{\Psi}_{i}$ is the standard basis function of the lowest order Nédélec finite element. Here $x$ and $y$ correspond to the degrees of freedom of $\operatorname{Re} \mathbf{A}_{h}$ and $\operatorname{Im} \mathbf{A}_{h}$ respectively.

Lemma 4.1. Let $K, M \in \mathbb{R}^{n \times n}$ be symmetric and semi-positive definite matrices such that $K+M$ is invertible. Define

$$
A=\left(\begin{array}{cc}
K & -M \\
-M & -K
\end{array}\right), \quad B=\left(\begin{array}{cc}
K+M & 0 \\
0 & K+M
\end{array}\right)
$$

Then the condition number of $B^{-1} A$ satisfies $\kappa\left(B^{-1} A\right) \leq \sqrt{2}$.

Proof. Let $\sigma\left(B^{-1} A\right)$ be the set of eigenvalues of $B^{-1} A$. Let $\lambda \in \sigma\left(B^{-1} A\right)$ and $z=\left(\begin{array}{l}x \\ y\end{array}\right)$, where $x, y \in \mathbb{R}^{n}$, be the corresponding eigenvector. It is easy to see that

$$
z^{T} B z=x^{T}(K+M) x+y^{T}(K+M) y
$$

and, by Cauchy-Schwarz inequality,

$$
\left|z^{T} A z\right|=\left|x^{T} K x-x^{T} M y-y^{T} M x-y^{T} K y\right| \leq z^{T} B z .
$$

It follows from $A z=\lambda B z$ that

$$
|\lambda|=\frac{\left|z^{T} A z\right|}{\left|z^{T} B z\right|} \leq 1, \quad \forall \lambda \in \sigma\left(B^{-1} A\right) .
$$

To derive a lower bound of $|\lambda|$, we set $z_{1}=\left(\begin{array}{c}x \\ -y\end{array}\right), z_{2}=\left(\begin{array}{c}-y \\ -x\end{array}\right) \in \mathbb{R}^{2 n}$. It is easy to check that

$$
z_{1}^{T} A z=x^{T} K x+y^{T} K y, \quad z_{2}^{T} A z=x^{T} M x+y^{T} M y
$$

which yield $\left(z_{1}+z_{2}\right)^{T} A z=z^{T} B z$. Moreover, we have

$$
\begin{aligned}
\left(z_{1}+z_{2}\right)^{T} B\left(z_{1}+z_{2}\right) & =(x-y)^{T}(K+M)(x-y)+(-x-y)^{T}(K+M)(-x-y) \\
& =2 z^{T} B z
\end{aligned}
$$

Therefore, by using $A z=\lambda B z$ again we obtain

$$
\frac{1}{|\lambda|}=\frac{\left|\left(z_{1}+z_{2}\right)^{T} B z\right|}{\left|\left(z_{1}+z_{2}\right)^{T} A z\right|} \leq \frac{\sqrt{\left(z_{1}+z_{2}\right)^{T} B\left(z_{1}+z_{2}\right)}}{\sqrt{z^{T} B z}} \leq \sqrt{2}, \quad \forall \lambda \in \sigma\left(B^{-1} A\right) .
$$

This completes the proof. $\square$

If $\sigma>0$ in the whole domain $\Omega, K+M$ is invertible. Lemma 4.1 suggests to choose the preconditioning matrix for (4.1) as

$$
\left(\begin{array}{cc}
K+M & 0 \\
0 & K+M
\end{array}\right)^{-1}
$$


That is, the preconditioning matrix $K+M$ is chosen as the finite element discretization of the following problem

$$
\begin{array}{rlrl}
\nabla \times \nabla \times \mathbf{A}+s^{2} \omega \sigma \mu \mathbf{A} & =\mathbf{f} & & \text { in } \Omega, \\
\mathbf{A} \times \mathbf{n}=0 & & \text { on } \Gamma .
\end{array}
$$

This preconditioning discrete problem can be solved efficiently by the preconditioned CG method with the Hiptmair-Xu auxiliary space preconditioner [16] (AMS/PCG). We use the implementation in hypre [18] which works also for partly vanishing conductivity $\sigma$ as in our situation in which case $(K+M)^{-1}$ should be understood as the inverse in the subspace that $K+M$ is invertible.

Through numerical experiments we found that with the preconditioned MINRES method the preconditioning system needs to be solved "exactly" in order to ensure convergence, while with the preconditioned GMRES method the preconditioning system can be solved approximately, resulting in shorter overall computing time. Thus the preconditioned GMRES method was used in the numerical examples presented here in which the preconditioning system was approximately solved with a few AMS/PCG iterations such that the residual of the preconditioning system was reduced by a factor of 0.01 . Typically $6-8 \mathrm{AMS} / \mathrm{PCG}$ iterations were performed for solving each preconditioning system in our numerical examples.

In practical applications, the impedance on the electrodes is of particular interest. The impedance on the electrode $S_{j}$ is defined as

$$
Z_{j}=R_{j}+\mathbf{i} \omega L_{j}=\frac{V}{I_{j}}
$$

where $V$ is the voltage, $\omega=2 \pi f$ is the angular frequency with the frequency $f$, and $I_{j}$ is the total current on the electrode $S_{j}$

$$
I_{j}=\int_{S_{j}} \sigma \mathbf{E} \cdot \mathbf{n} d s=\int_{S_{j}} \sigma\left(-\mathbf{i} \omega \mathbf{A}-s^{-1} \nabla \phi_{0}\right) \cdot \mathbf{n} d s .
$$

$R_{j}$ and $L_{j}$ are respectively the usual electric resistance and inductance.

4.1. Example 1. In this example, we consider the parasitic parameters of a straight conductor as described in Figure 4.1. The setting of the problem is as follows: $\sigma=5.8 \times 10^{7} \mathrm{~S} / \mathrm{m}, \mu=\mu_{0}=4 \pi \times 10^{-7} \mathrm{H} / \mathrm{m}$, the size of the conductor is $1 \times 1 \times 5(\mu \mathrm{m})$. With this size, the scaling factor $s=10^{-6}$. The computing domain is $\Omega=[0,5]^{3}$, $S_{1}=0 \times[2,3] \times[2,3], S_{2}=5 \times[2,3] \times[2,3]$. We set the voltage to be $1 \mathrm{~V}$, i.e., $\left.\phi_{0}\right|_{S_{1}}=1,\left.\phi_{0}\right|_{S_{2}}=0$. There is no source current, $\mathbf{J}_{s}=0$.

For DC (Direct Current) circuit, the current density is uniformly distributed on the cross section of the conductor, in the case of straight conductor, the resistance can be calculated by the Ohm law (4.2)

$$
R=\frac{1}{\sigma} \frac{l}{S}
$$

where $l$ is the length of the conductor, $S$ is the cross section area of the conductor.

It is well-known that for the AC (Alternating Current) circuit, the current is concentrated on the surface of the conductor, the so-called skin effect. Skin effect causes the decrease of the virtual conductive cross section. The skin-depth is defined as

$$
\delta=\sqrt{\frac{2}{\omega \mu \sigma}}
$$




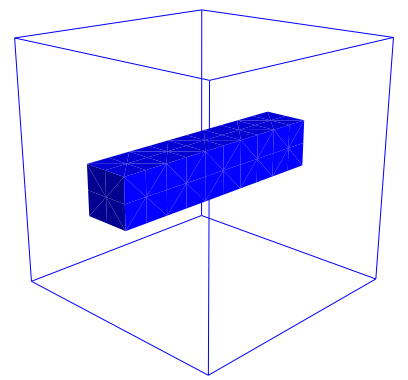

FIG. 4.1. The conductor and the computational domain (Example 1).

\begin{tabular}{|c|c|c|c|}
\hline Frequency $(\mathrm{Hz})$ & Skin-depth $(\mathrm{m})$ & $\operatorname{Re}(\mathrm{I})$ & $\operatorname{Im}(\mathrm{I})$ \\
\hline $10^{1}$ & $2.089807 \mathrm{e}-02$ & $1.160086 \mathrm{e}+01$ & $5.623839 \mathrm{e}-04$ \\
\hline $10^{2}$ & $6.608549 \mathrm{e}-03$ & $1.160000 \mathrm{e}+01$ & $-2.721970 \mathrm{e}-05$ \\
\hline $10^{3}$ & $2.089807 \mathrm{e}-03$ & $1.160000 \mathrm{e}+01$ & $4.437668 \mathrm{e}-06$ \\
\hline $10^{4}$ & $6.608549 \mathrm{e}-04$ & $1.160000 \mathrm{e}+01$ & $-1.547581 \mathrm{e}-05$ \\
\hline $10^{5}$ & $2.089807 \mathrm{e}-04$ & $1.160000 \mathrm{e}+01$ & $-1.493227 \mathrm{e}-04$ \\
\hline $10^{6}$ & $6.608549 \mathrm{e}-05$ & $1.160000 \mathrm{e}+01$ & $-1.493697 \mathrm{e}-03$ \\
\hline $10^{7}$ & $2.089807 \mathrm{e}-05$ & $1.159998 \mathrm{e}+01$ & $-1.493666 \mathrm{e}-02$ \\
\hline $10^{8}$ & $6.608549 \mathrm{e}-06$ & $1.159805 \mathrm{e}+01$ & $-1.493417 \mathrm{e}-01$ \\
\hline $10^{9}$ & $2.089807 \mathrm{e}-06$ & $1.140516 \mathrm{e}+01$ & $-1.485203 \mathrm{e}+00$ \\
\hline $10^{10}$ & $6.608549 \mathrm{e}-07$ & $4.322117 \mathrm{e}+00$ & $-5.564086 \mathrm{e}+00$ \\
\hline $10^{11}$ & $2.089807 \mathrm{e}-07$ & $1.133206 \mathrm{e}-01$ & $-9.264290 \mathrm{e}-01$ \\
\hline
\end{tabular}

The skin-depth, the real and imaginary part of the current, $\operatorname{Re}(\mathrm{I}), \operatorname{Im}(\mathrm{I})$ (Example 1).

that is, the virtual conductive part is concentrated in the layer of thickness $\delta$ from the surface.

For our first example, by (4.2), we can calculate that the $\mathrm{DC}$ resistance is 0.086207 $\mathrm{Ohm}$, and the current is $11.6 \mathrm{~A}$. Table 4.1 shows the skin-depth, the real and imaginary part of the current for different choices of frequencies. We observe that in the case of low frequency, numerical results match the DC value very well.

Figures 4.2 and 4.3 show the resistance and inductance for the frequency from $1 \mathrm{GHz}$ to $100 \mathrm{GHz}$ where the results computed by the FastImp method [33] are also displayed. We observe that for both our method and the FastImp method the resistance increases and the inductance decreases as the frequency increases. From Figure 4.2 , we can also observe that, at low frequency, both results are very close to the DC resistance. By Table 4.1, we know that when the frequency increases to $10 \mathrm{GHz}$, the skin-depth is less than the size of the cross section of the conductor. Increasing the frequency, the skin-depth becomes smaller, the resistance becomes larger. We remark that the FastImp calculation is based on the integral method whose underlying mathematical model is different from the one used in this paper.

Figure 4.4 shows the mesh generated by our adaptive method for two different choices of frequency. Figure 4.5 shows the corresponding current density. The meshes and current density match the skin-depth in Table 4.1 very well.

4.2. Example 2. This example concerns the L-shaped conductor as shown in Figure 4.6. The cross-section of the L-shaped conductor is $1 \times 1 \mu \mathrm{m}$ and the compu- 


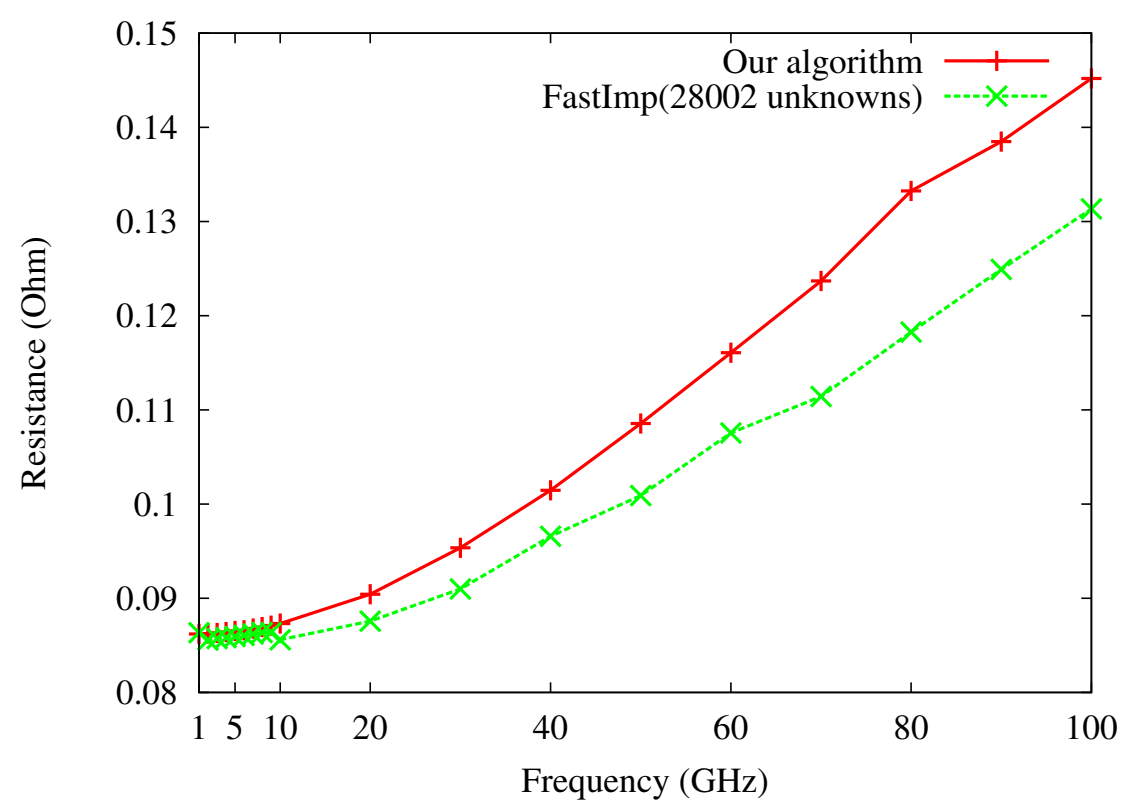

FIG. 4.2. The relation between the resistance and the frequency (Example 1).

tational domain is $5 \times 5 \times 5 \mu \mathrm{m}$. The material parameters are the same as those in Example 1.

Figures 4.7 and 4.8 show the inductance and the resistance for different choices of the frequency where the results from FastImp are again displayed. We can still observe the phenomenon that the resistance becomes larger and the inductance becomes smaller when we increasing the frequency. And the skin effect governs the turning point of the resistance, too.

Figures $4.9,4.10,4.11$ show the $\log N-\log \mathcal{E}$ curves for different choices of frequencies, where $\mathcal{E}$ is the a posteriori error estimate and $N$ is the number of degrees of freedom. They indicate that the meshes and the associated numerical complexity are quasi-optimal: $\mathcal{E} \approx C N^{-1 / 3}$ is valid asymptotically for our adaptive algorithm, but invalid for uniform refinement.

Figure 4.12 shows a sample of the mesh on the plane $z=0.25$ and Figure 4.13 shows the current density on that plane when the frequency is $100 \mathrm{GHz}$. We observe our method captures the skin effect and the singularity of the solution rather well.

Table 4.2 shows the relative error of the resistance $R R$, the relative error of the inductance $R L$, the number of the GMRES iterations and the time required to reduce the initial residual by a factor $10^{-10}$ for solving the linear system of equations when using adaptive mesh refinements. Table 4.3 shows the results using uniform mesh refinements. The relative error of the resistance $R R$ is defined as $R R=|R-\hat{R}| /|\hat{R}|$, where $R$ is the resistance and $\hat{R}$ is the resistance at the last adaptive refinement step which we take as the "exact" solution. The relative error of the inductance $R L$ is defined similarly. We observe that for the resistance, the numerical result with 147,176 degrees of freedom with adaptive mesh refinements is similar to the result with 1,821,040 degrees of freedom using uniform mesh refinements, that is, the relative error is less than $1 \%$. This demonstrates clearly the efficiency of our adaptive 


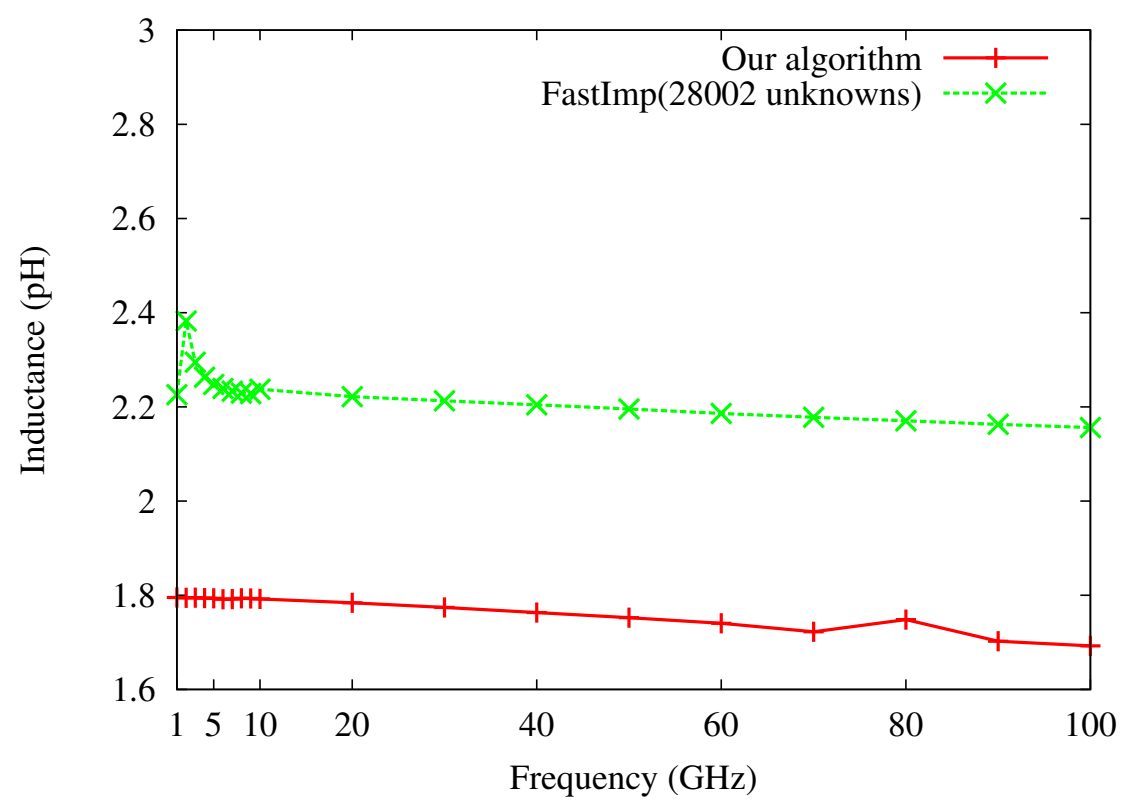

FIG. 4.3. The relation between the inductance and the frequency (Example 1).
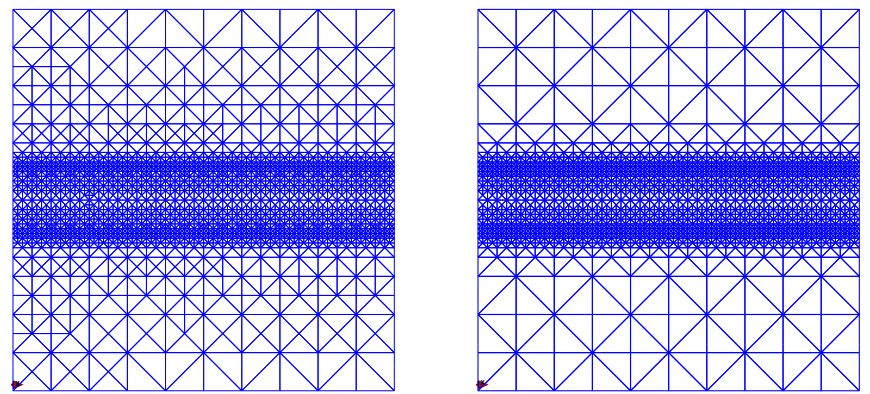

FIG. 4.4. Meshes generated by the adaptive algorithm. Left: $f=10 \mathrm{GHz}$, Right: $f=100 \mathrm{GHz}$ (Example 1).
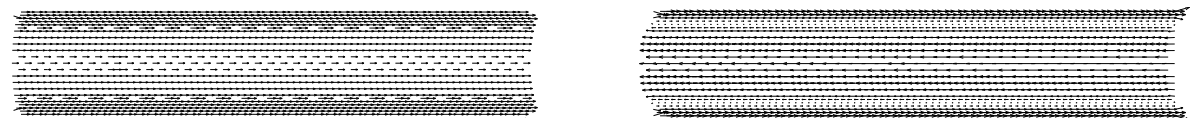

FIG. 4.5. The current density $\mathbf{J}$ in the conductor. Left: $f=10 \mathrm{GHz}$, Right: $f=100 \mathrm{GHz}$ (Example 1).

algorithm.

The fourth column of the tables shows the numbers of GMRES iterations required to reduce the initial residual by a factor $10^{-10}$. The stable iteration numbers in Tables 4.2 and 4.3 indicate that the preconditioner is optimal.

4.3. Example 3. In this example, the material parameters of the conductors are the same as those in Example 1. The frequency is 1Ghz, 10Ghz and 100Ghz, the size of each conductor's cross section is $1 \times 1(\mu \mathrm{m})$, the computational domain 


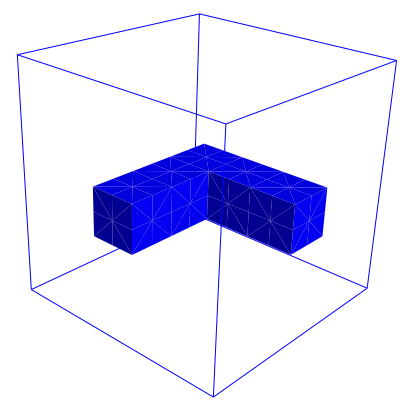

FIG. 4.6. The conductor and the computational domain (Example 2).

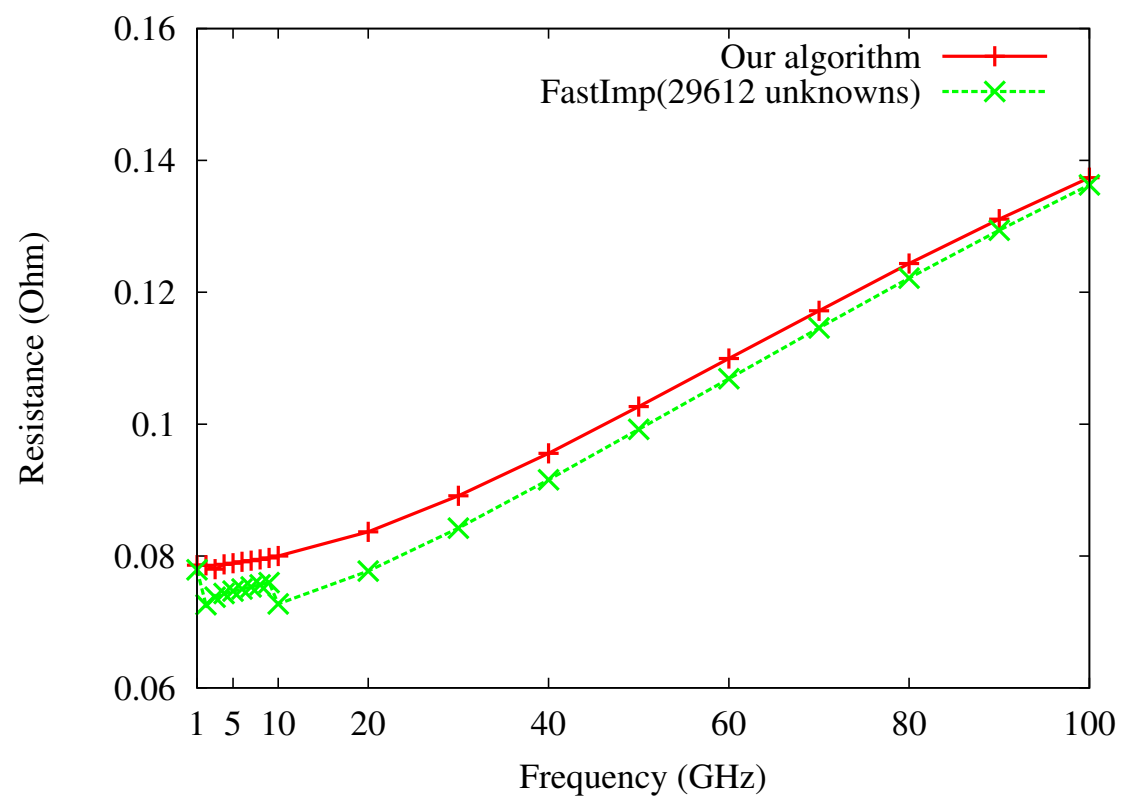

FIG. 4.7. The relation between the resistance and the frequency (Example 2).

is $9 \times 9 \times 5(\mu \mathrm{m})$, and the distance between two neighbor conductor is $0.5 \mu \mathrm{m}$. The conductors and computational domain are showed in Figure 4.14. 1V voltage is fed on the electrode 3 .

Figures $4.15,4.16,4.17$ show the $\log N-\log \mathcal{E}$ curves for different choices of frequencies, where $\mathcal{E}$ is the a posteriori error estimate and $N$ is the number of degrees of freedom. They indicate that the meshes and the associated numerical complexity are quasi-optimal: $\mathcal{E} \approx C N^{-1 / 3}$ is valid asymptotically for our adaptive algorithm, but invalid for uniform refinement.

Figure 4.18 shows the mesh generated by the adaptive algorithm on the plane $x=0.4$. we find that the adaptive method can capture the singularities of the solution well.

In Tables 4.4 and 4.5 , we report the real part of the eddy current in the middle conductor on which $1 \mathrm{~V}$ voltage is fed. We compare the computational results of adaptive mesh refinements and uniform mesh refinements. we observe that the results 


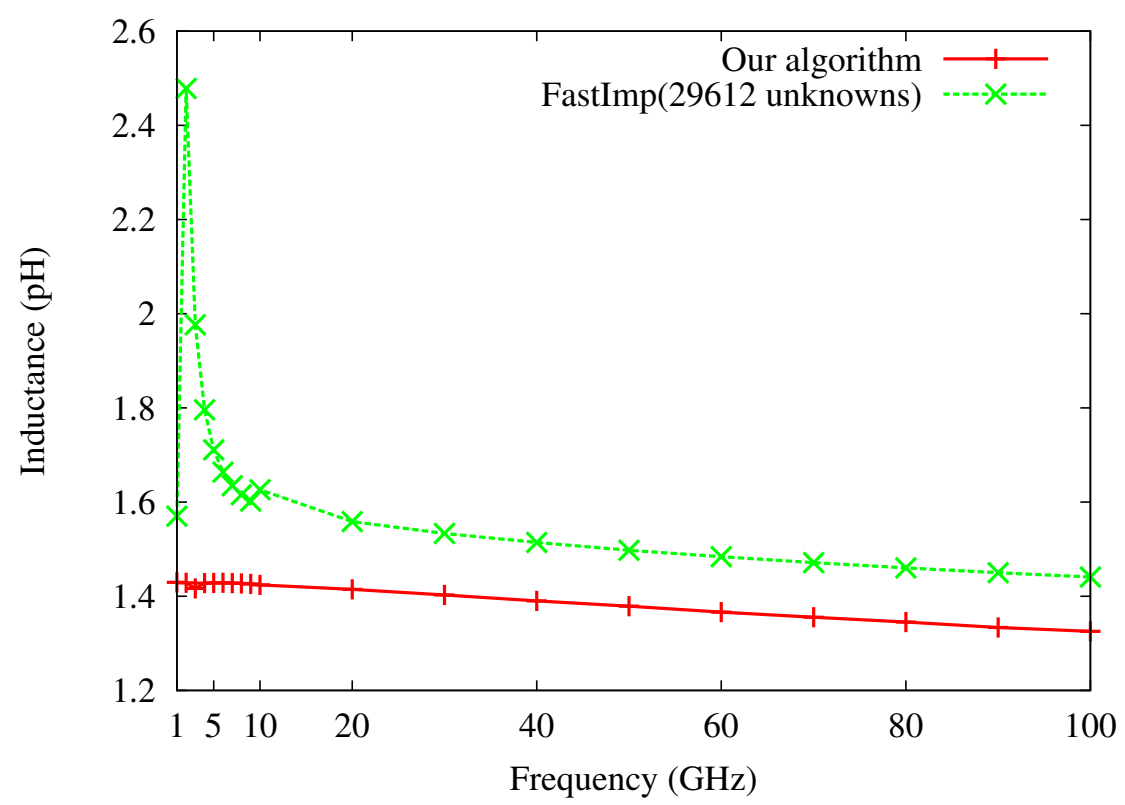

FIG. 4.8. The relation between the inductance and the frequency (Example 2).

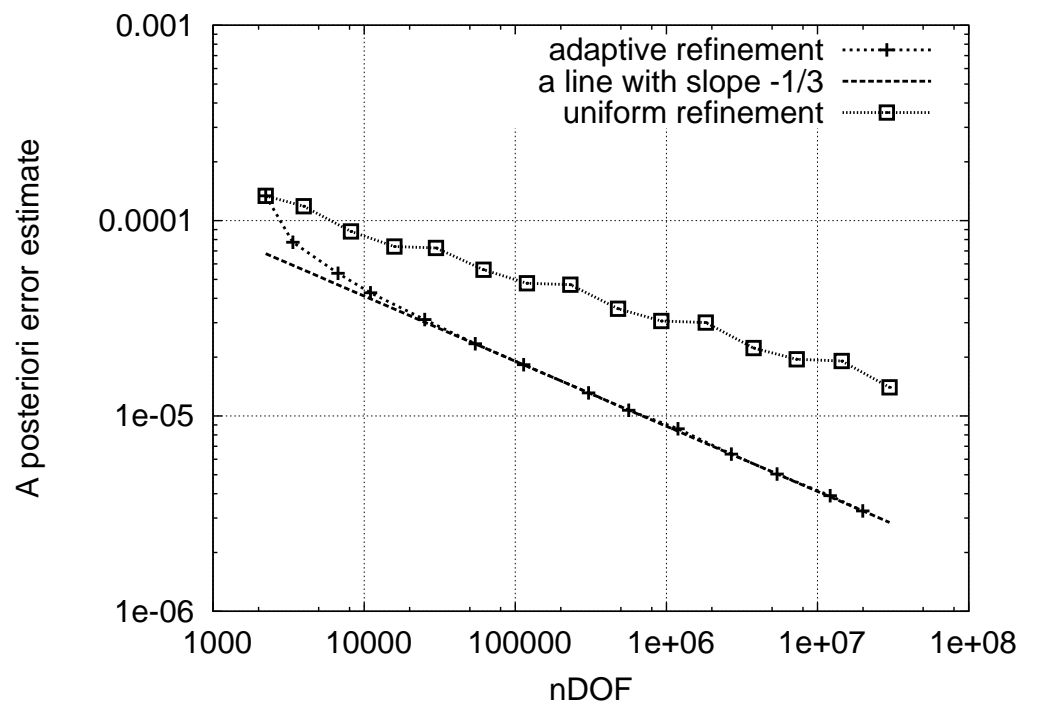

FIG. 4.9. The quasi-optimality of the adaptive mesh refinements. The frequency $f=1 \mathrm{GHz}$ (Example 2).

of adaptive mesh refinements with 633,004 DOF are similar to those of uniform mesh refinements with $35,048,800$ DOF. This comparison shows the excellent efficiency of our adaptive method.

Acknowledgement. The authors would like to thank Ralf Hiptmair from ETH, Qiya $\mathrm{Hu}$ from Chinese Academy of Sciences, Ulrich Langer from Johannes Kepler University Linz, Xuan Zeng from Fudan Univeristy for inspiring discussions. The 


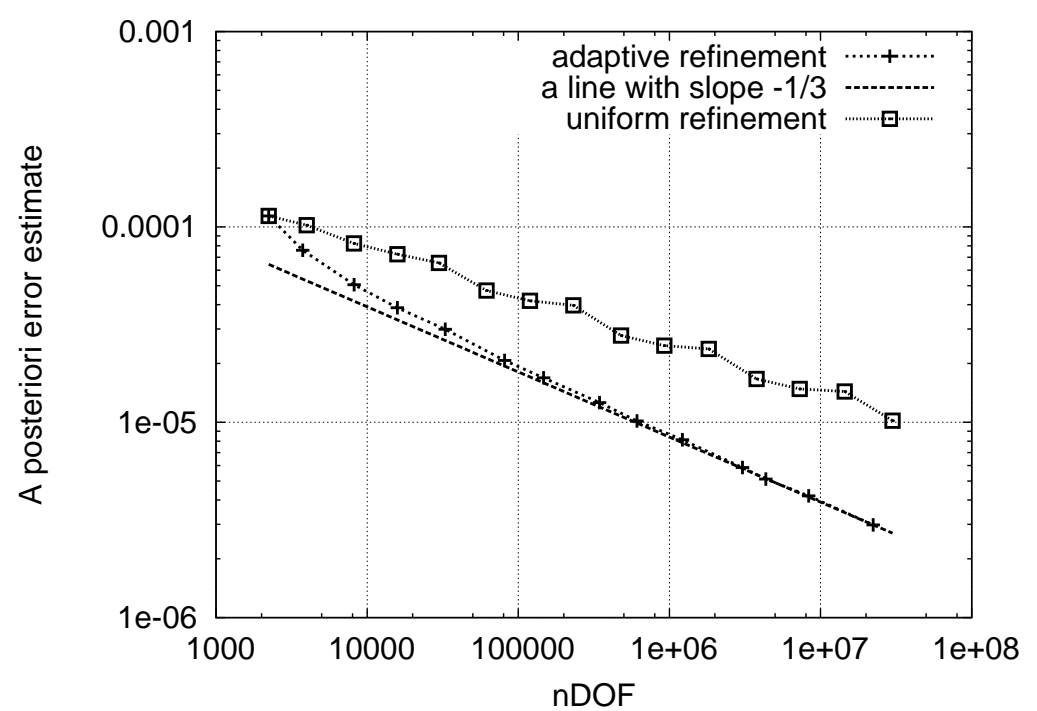

FIG. 4.10. The quasi-optimality of the adaptive mesh refinements. The frequency $f=10 \mathrm{GHz}$ (Example 2).

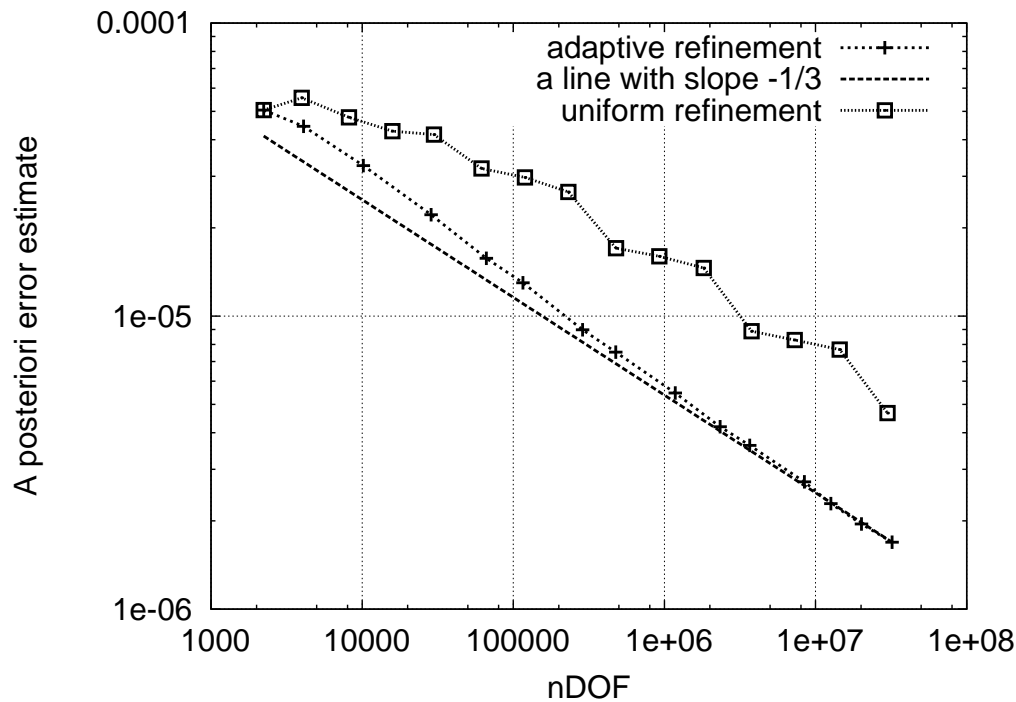

FIG. 4.11. The quasi-optimality of the adaptive mesh refinements. The frequency $f=100 \mathrm{GHz}$ (Example 2).

authors are also grateful to the anonymous referees for their constructive comments which, in particular, lead to Lemma 4.1.

\section{REFERENCES}

[1] C. Amrouche, C. Bernardi, M. Dauge and V. Girault, Vector potentials in three-dimensional non-smooth domains, Math. Meth. Appl. Sci. 21 (1998), 823-864. 


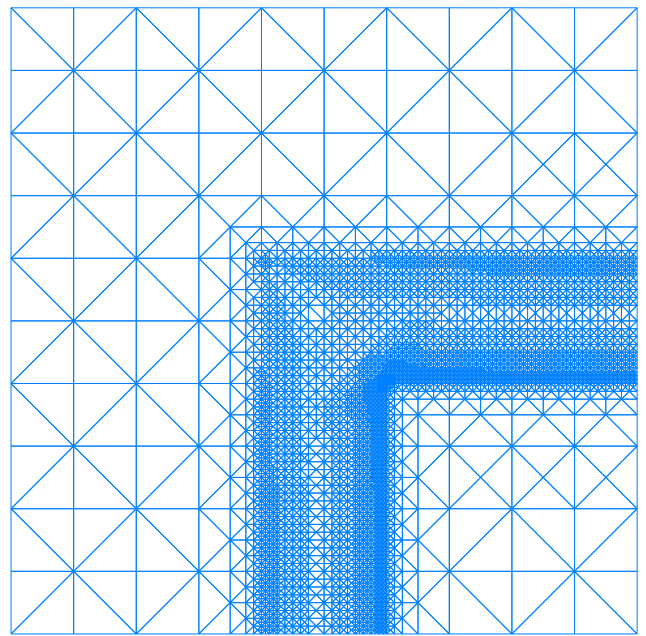

FIG. 4.12. The adaptive mesh on the plane $z=0.25$ with 3,044,344 degrees of freedom. The frequency $f=100$ Ghz (Example 2).

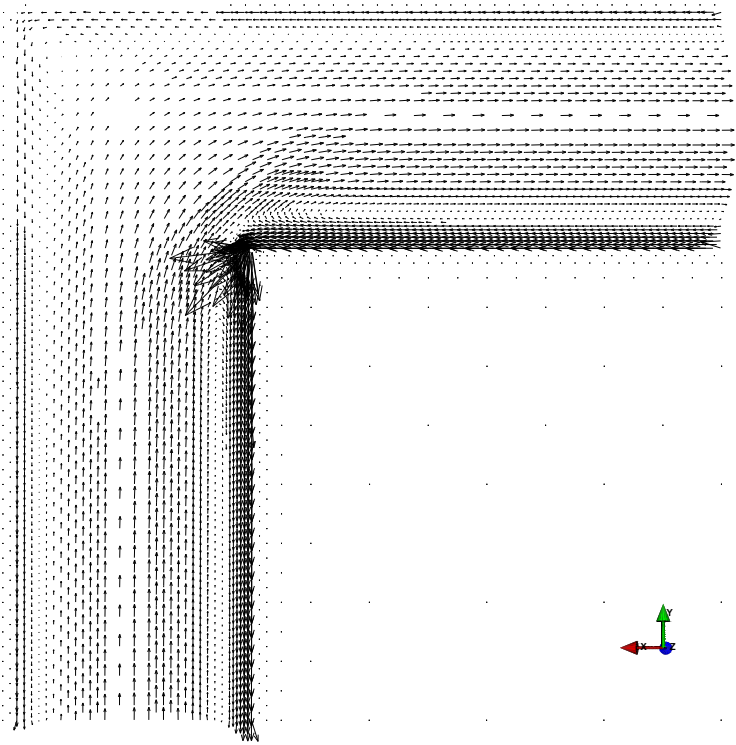

FIG. 4.13. The current density on the plane $z=0.25$. The frequency $f=100 \mathrm{GHz}$ (Example 2).

[2] F. Brezzi and M. Fortin, Mixed and Hybrid Finite Element Methods, Springer-Verlag, New York, 1991.

[3] V. Girault and P. A. Raviart, Finite Element Methods for Navier-Stokes Equations, SpringerVerlag, Berlin, 1980.

[4] R. Beck, R. Hiptmair, R. Hoppe and B. Wohlmuth, Residual based a posteriori error estimators for eddy current computation, Math. Model. Numer. Anal. 34 (2000), 159-182.

[5] A. Bermudez, R. Rodriguez and P. Salgado, Numerical analysis of electric field formulations of the eddy current model, Numer. Math. 102 (2005), 181-201.

[6] M.Sh. Birman and M.Z. Solomyak, $L^{2}$-Theory of the Maxwell operator in arbitary domains, Uspekhi Mat. Nauk 42 (1987), 61-76 (in Russian); Russian Math. Surveys 43 (1987), 75-96 


\begin{tabular}{|c|c|c|c|c|}
\hline DOF & $R R_{k}(\%)$ & $R L_{k}(\%)$ & Iterations & Time \\
\hline 2230 & 8.67038 & 11.3565 & 20 & $3.2210 \mathrm{~s}$ \\
\hline 3744 & 16.0901 & 3.82451 & 19 & $3.7475 \mathrm{~s}$ \\
\hline 8204 & 7.06066 & 0.158021 & 18 & $5.6478 \mathrm{~s}$ \\
\hline 15844 & 4.46469 & 0.58095 & 18 & $6.9424 \mathrm{~s}$ \\
\hline 32822 & 2.06267 & 1.64549 & 18 & $9.2179 \mathrm{~s}$ \\
\hline 81140 & 1.12457 & 1.974 & 18 & $13.2925 \mathrm{~s}$ \\
\hline 147176 & 0.757294 & 1.50113 & 18 & $16.0653 \mathrm{~s}$ \\
\hline 344864 & 0.410913 & 1.17031 & 18 & $21.9584 \mathrm{~s}$ \\
\hline 610396 & 0.284501 & 0.76923 & 18 & $31.6766 \mathrm{~s}$ \\
\hline 1218282 & 0.167636 & 0.397854 & 18 & $44.2505 \mathrm{~s}$ \\
\hline 3044344 & 0.0674971 & 0.306796 & 18 & $81.9320 \mathrm{~s}$ \\
\hline 4344878 & 0.0466162 & 0.139459 & 18 & $108.1488 \mathrm{~s}$ \\
\hline 8352352 & 0.0420997 & 0.0631803 & 18 & $192.1478 \mathrm{~s}$ \\
\hline 22299274 & - & - & 29 & $1554.1333 \mathrm{~s}$ \\
\hline
\end{tabular}

The relative error of the resistance, the relative error of the inductance, the number of GMRES iterations and the time required to reduce the initial residual by a factor $10^{-10}$ in the case of adaptive mesh refinements (48-cpu). The "exact" solution is $\hat{R}=7.995037 \times 10^{-2}(\mathrm{Ohm}), \hat{L}=1.427386 \times$ $10^{-3}(\mathrm{nH})$. The frequency $f=10 \mathrm{GHz}$ (Example 2).

\begin{tabular}{|c|c|c|c|c|}
\hline DOF & $R R_{k}(\%)$ & $R L_{k}(\%)$ & Iterations & Time \\
\hline 2230 & 8.67038 & 11.3565 & 20 & $3.0062 \mathrm{~s}$ \\
\hline 3980 & 17.0202 & 2.42368 & 20 & $3.6666 \mathrm{~s}$ \\
\hline 8180 & 17.7352 & 5.30035 & 19 & $4.7251 \mathrm{~s}$ \\
\hline 15860 & 17.3 & 8.02768 & 19 & $6.3077 \mathrm{~s}$ \\
\hline 29860 & 9.05916 & 2.36667 & 19 & $7.1054 \mathrm{~s}$ \\
\hline 61660 & 5.18414 & 1.02826 & 19 & $9.7042 \mathrm{~s}$ \\
\hline 119320 & 5.25714 & 1.91719 & 18 & $12.4787 \mathrm{~s}$ \\
\hline 231320 & 2.59927 & 0.429164 & 18 & $15.6587 \mathrm{~s}$ \\
\hline 478520 & 1.50422 & 0.256784 & 18 & $20.8916 \mathrm{~s}$ \\
\hline 925040 & 1.40578 & 0.37656 & 18 & $37.8598 \mathrm{~s}$ \\
\hline 1821040 & 0.778187 & 0.0200328 & 18 & $59.3284 \mathrm{~s}$ \\
\hline 3769840 & 0.415279 & 0.127481 & 18 & $101.0462 \mathrm{~s}$ \\
\hline 7283680 & 0.420746 & 0.0914783 & 18 & $201.6271 \mathrm{~s}$ \\
\hline 14451680 & 0.253912 & 0.0883963 & 18 & $426.5594 \mathrm{~s}$ \\
\hline 29926880 & 0.106532 & 0.394561 & 18 & $944.9077 \mathrm{~s}$ \\
\hline
\end{tabular}

The relative error of the resistance, the relative error of the inductance, the number of GMRES iterations and the time required to reduce the initial residual by a factor $10^{-10}$ in the case of uniform mesh refinements (48-cpu). The "exact" solution is $\hat{R}=7.995037 \times 10^{-2}$ (Ohm), $\hat{L}=1.427386 \times$ $10^{-3}(\mathrm{nH})$. The frequency $f=10 \mathrm{GHz}$ (Example 2).

(in English).

[7] A. Bossavit, Computational Electromagnetism: Variational Formulation, Complementarity, Edge Elements, in Academic Press Electromagnetism Series, no.2, Academic Press, San Diego, 1998.

[8] A. Buffa, M. Costabel and D. Sheen. On traces for $H(\mathbf{c u r l}, \Omega)$ in Lipschitz domain, J. Math. Anal. Appl. 276 (2002), 845-876. 

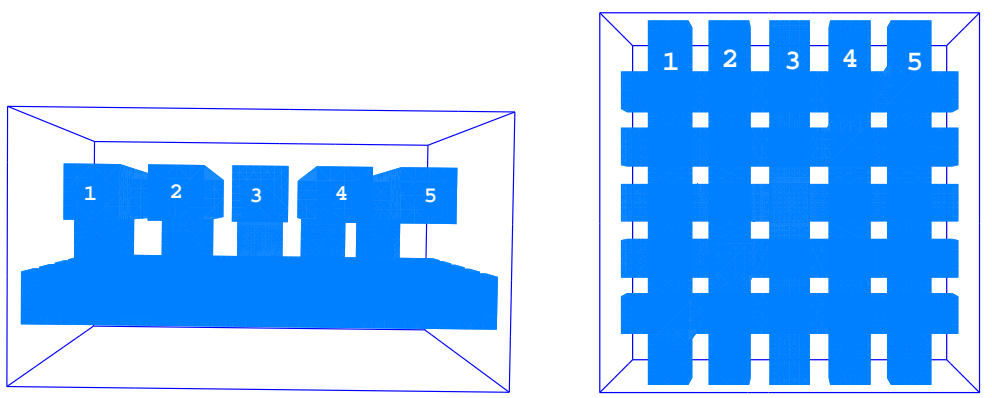

FIG. 4.14. Side view (left) and plan view (right) of the conductors and the computational domain (Example 3).

Log-log plot of A Posterior error w.r.t. Degrees of Freedom $(1 \mathrm{GHz})$

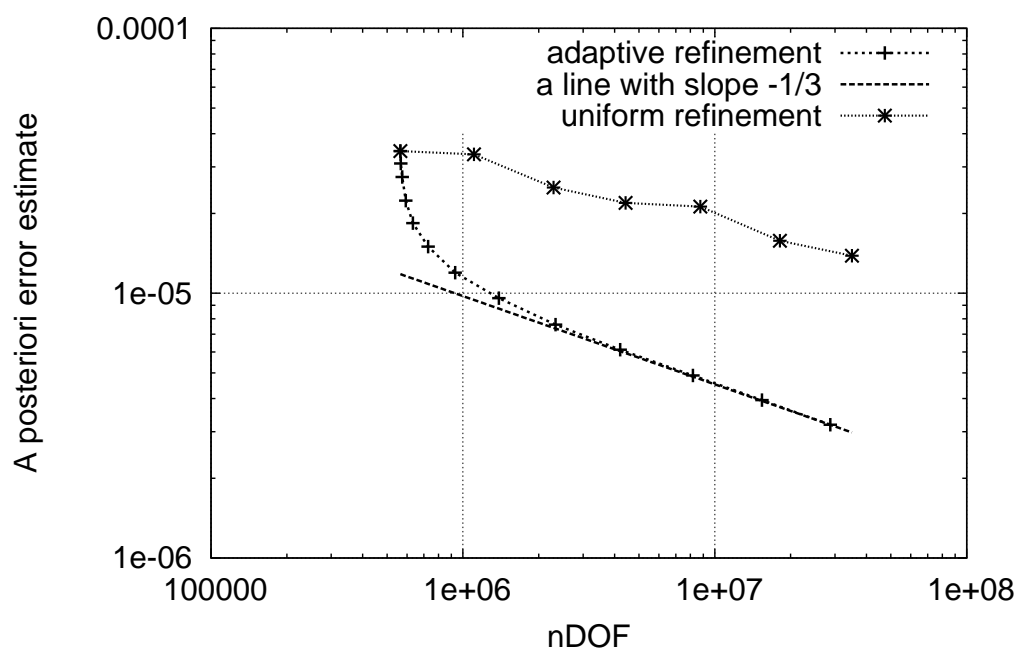

FIG. 4.15. The quasi-optimality of the adaptive mesh refinements. The frequency $f=1 \mathrm{GHz}$ (Example 3).

[9] M. Cessenat, Mathematical Methods in Electromagnetism, Linear Theory and Applications, World Scientific, Singapore, 1996.

[10] J. Chen and Z. Chen, An adaptive perfectly matched layer technique for 3-D time-harmonic electromagnetic scattering problems, Math. Comp, 77 (2008), 673-698.

[11] Z. Chen, Q. Du and J. Zou, Finite element methods with matching and nonmatching meshes for maxwell equations with discontinuous coefficients, SIAM J. Numer. Anal. 37 (2000), 1542-1570.

[12] Z. Chen, L. Wang and W. Zheng, An adaptive multilevel method for time-harmonic Maxwell equations with singularities, SIAM J. Sci. Comput. 29 (2007), 118-138.

[13] P. Dular, Dual magnetodynamic finite element formulations with natural definitions of global quantities for electric circuit coupling, In U. van Rienen, M. Günther, and D. Hecht, eds, Scientific Computing in Electrical Engineering. Lecture Notes in Computer Science and Engineering, Vol. 18, 367-378, Springer, Berlin, 2001.

[14] H. Heeb and A.E. Ruehli, Three-dimensional interconnect analysis using partial element equivalent circuits, IEEE Trans. Circuits Sys.-I: Fundamental Theory Appl. 39 (1992), 974-982.

[15] R. Hiptmair and O. Sterz, Current and voltage excitations for the eddy current model, Int. J. Numer. Modelling 18 (2005), 1-21.

[16] R. Hiptmair and J. Xu, Nodal auxiliary space preconditioning in $H($ curl $)$ and $H($ div $)$ spaces, Research Report No. 2006-09, Seminar für Angewandte Mathematik, Eidgenössische Tech- 
Log-log plot of A Posterior error w.r.t. Degrees of Freedom $(10 \mathrm{GHz})$

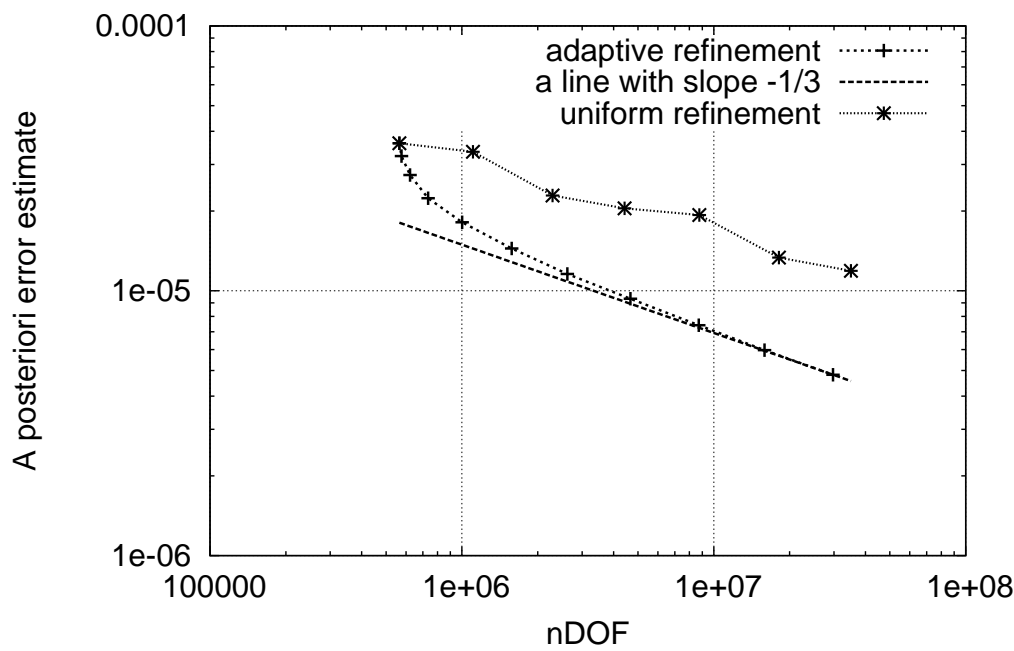

FIG. 4.16. The quasi-optimality of the adaptive mesh refinements. The frequency $f=10 \mathrm{GHz}$ (Example 3).

Log-log plot of A Posterior error w.r.t. Degrees of Freedom $(100 \mathrm{GHz})$

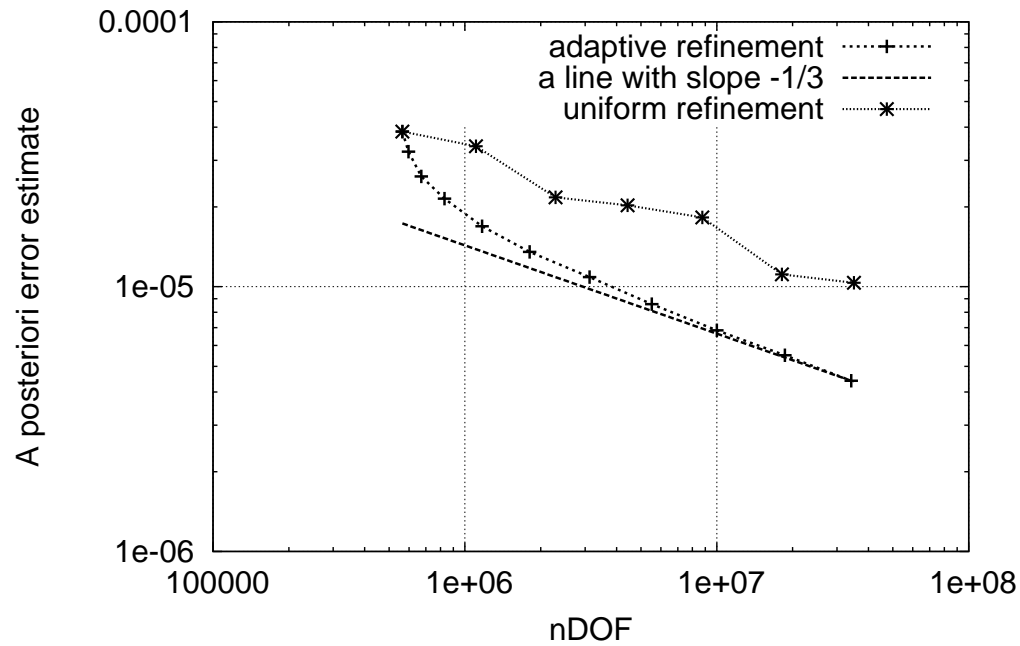

FIG. 4.17. The quasi-optimality of the adaptive mesh refinements. The frequency $f=100 \mathrm{GHz}$ (Example 3).

nische Hochschule, CH-8092 Zürich, Switzerland, May 2006.

[17] L. Kettunen, Fields and circuits in computational electromagnetism, IEEE Trans. Magetics 37 (2001), 3393-3396.

[18] hypre: High performance preconditioners, http://www.llnl.gov/CASC/hypre/.

[19] M. Kamon, M.J. Tsuk and J.K. White, FASTHENRY: A multipole-accelerated 3-D inductance extraction program, IEEE Trans. Micro. Theory Tech. 42 (1994), 1750-1758.

[20] Tz.V. Kolev and P.S. Vassilevski, Some experience with a H1-based auxiliary space AMG for H(curl) problems, LLNL Technical Report UCRL-TR-221841, June, 2006.

[21] Tz.V. Kolev and P.S. Vassilevski, Parallel auxiliary space AMG for H(curl) problems, J. Com- 


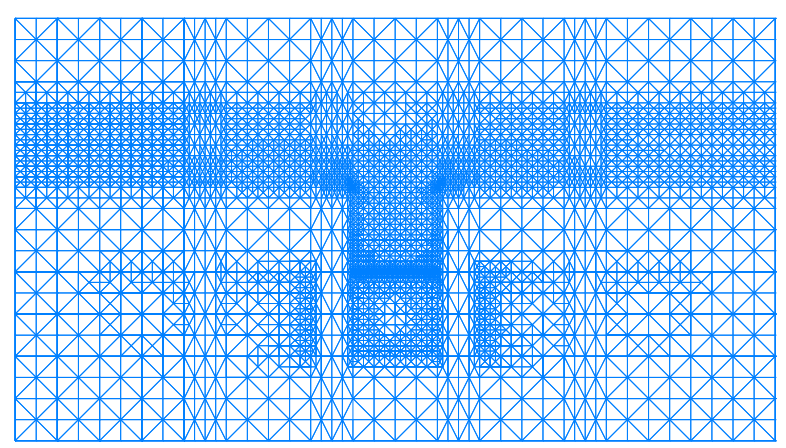

FIG. 4.18. The adaptive mesh on the plane $x=0.4$ with the degrees of freedom $2,331,652$. The frequency $f=1 G h z$.

\begin{tabular}{|c|c|c|c|c|}
\hline \multicolumn{7}{|c|}{ Conductor 3 } \\
\hline DOFs & electrode 1 & electrode 2 & electrode 3 & electrode 4 \\
\hline 564472 & $-9.004836 \mathrm{e}+00$ & $3.877742 \mathrm{e}+00$ & $2.563490 \mathrm{e}+00$ & $2.563589 \mathrm{e}+00$ \\
\hline 566554 & $-8.987716 \mathrm{e}+00$ & $3.874469 \mathrm{e}+00$ & $2.556535 \mathrm{e}+00$ & $2.556709 \mathrm{e}+00$ \\
\hline 574510 & $-8.975981 \mathrm{e}+00$ & $3.878613 \mathrm{e}+00$ & $2.548175 \mathrm{e}+00$ & $2.548257 \mathrm{e}+00$ \\
\hline 593158 & $-8.964733 \mathrm{e}+00$ & $3.879230 \mathrm{e}+00$ & $2.542745 \mathrm{e}+00$ & $2.542818 \mathrm{e}+00$ \\
\hline 633004 & $-8.958545 \mathrm{e}+00$ & $3.881884 \mathrm{e}+00$ & $2.538108 \mathrm{e}+00$ & $2.538157 \mathrm{e}+00$ \\
\hline 727668 & $-8.954078 \mathrm{e}+00$ & $3.882076 \mathrm{e}+00$ & $2.535473 \mathrm{e}+00$ & $2.535529 \mathrm{e}+00$ \\
\hline 931448 & $-8.954033 \mathrm{e}+00$ & $3.883169 \mathrm{e}+00$ & $2.533677 \mathrm{e}+00$ & $2.533774 \mathrm{e}+00$ \\
\hline 1388802 & $-8.952550 \mathrm{e}+00$ & $3.884290 \mathrm{e}+00$ & $2.532588 \mathrm{e}+00$ & $2.532685 \mathrm{e}+00$ \\
\hline 2331652 & $-8.951816 \mathrm{e}+00$ & $3.885182 \mathrm{e}+00$ & $2.532959 \mathrm{e}+00$ & $2.533031 \mathrm{e}+00$ \\
\hline 4212272 & $-8.950144 \mathrm{e}+00$ & $3.885556 \mathrm{e}+00$ & $2.532418 \mathrm{e}+00$ & $2.532497 \mathrm{e}+00$ \\
\hline 8190290 & $-8.950675 \mathrm{e}+00$ & $3.885643 \mathrm{e}+00$ & $2.532367 \mathrm{e}+00$ & $2.532434 \mathrm{e}+00$ \\
\hline 15386978 & $-8.950395 \mathrm{e}+00$ & $3.885685 \mathrm{e}+00$ & $2.532283 \mathrm{e}+00$ & $2.532370 \mathrm{e}+00$ \\
\hline 28728986 & $-8.951584 \mathrm{e}+00$ & $3.885673 \mathrm{e}+00$ & $2.532178 \mathrm{e}+00$ & $2.532264 \mathrm{e}+00$ \\
\hline
\end{tabular}

The real part of the eddy current in the middle conductor using adaptive mesh refinements. The frequency is $1 \mathrm{GHz}$ (48-cpu).

\begin{tabular}{|c|c|c|c|c|}
\hline \multicolumn{7}{|c|}{ Conductor 3} \\
\hline DOFs & electrode 1 & electrode 2 & electrode 3 & electrode 4 \\
\hline 564472 & $-9.004836 \mathrm{e}+00$ & $3.877742 \mathrm{e}+00$ & $2.563490 \mathrm{e}+00$ & $2.563589 \mathrm{e}+00$ \\
\hline 1106552 & $-8.997516 \mathrm{e}+00$ & $3.878391 \mathrm{e}+00$ & $2.559477 \mathrm{e}+00$ & $2.559583 \mathrm{e}+00$ \\
\hline 2290328 & $-8.979082 \mathrm{e}+00$ & $3.879848 \mathrm{e}+00$ & $2.549454 \mathrm{e}+00$ & $2.549488 \mathrm{e}+00$ \\
\hline 4425776 & $-8.972148 \mathrm{e}+00$ & $3.882063 \mathrm{e}+00$ & $2.545029 \mathrm{e}+00$ & $2.545116 \mathrm{e}+00$ \\
\hline 8762416 & $-8.968144 \mathrm{e}+00$ & $3.882562 \mathrm{e}+00$ & $2.543162 \mathrm{e}+00$ & $2.543299 \mathrm{e}+00$ \\
\hline 18143920 & $-8.961368 \mathrm{e}+00$ & $3.883401 \mathrm{e}+00$ & $2.538966 \mathrm{e}+00$ & $2.539060 \mathrm{e}+00$ \\
\hline 35048800 & $-8.958832 \mathrm{e}+00$ & $3.884084 \mathrm{e}+00$ & $2.537322 \mathrm{e}+00$ & $2.537411 \mathrm{e}+00$ \\
\hline
\end{tabular}

The real part of the eddy current in the middle conductor using uniform mesh refinements. The frequency is $1 \mathrm{GHz}(48-\mathrm{cpu})$. 
put. Math. 27 (2009), 604-623.

[22] P. Monk, Finite Elements Methods for Maxwell Equations, Oxford University Press, Oxford, 2003.

[23] P. Monk, A posteriori error indicators for Maxwell's equations, J. Comp. Appl. Math. 100 (1998), 173-190

[24] J.C. Nédélec, Mixed finite elements in $\mathbf{R}^{3}$, Numer. Math. 35 (1980), 315-341.

[25] NETGEN, http://www.hpfem.jku.at/netgen/.

[26] PHG, Parallel Hiarachical Grid, http://lsec.cc.ac.cn/phg/.

[27] L.-B. Zhang, A Parallel algorithm for adaptive local refinement of tetrahedral meshes using bisection, Numerical Mathematics: Theory, Methods and Applications 2 (2009), 65-89.

[28] S. Reitzinger and J. Schöberl, An algebraic multigrid method for finite element discretizations with edge elements, Numer. Linear Algebra Appl. 9 (2002), 223-238.

[29] G. Rubinacci, A. Tamburrino and F. Villone, Circuits/Fields coupling and multiply connected domains in integral formulations, IEEE Trans. Magn. 38 (2002), 581-584.

[30] A.E. Ruehli, Equivalent circuit models for three-dimensional multiconductor systems, IEEE Trans. Microwave Theory Tech. 22 (1974), 216-221.

[31] L.R. Scott and S. Zhang, Finite element interpolation of nonsmooth functions satisfying boundary conditions, Math. Comp. (1990), 483-493.

[32] W. Zheng, Z. Chen and L. Wang, An adaptive finite element methods for the $H-\phi$ formulation of time-dependent eddy current problems, Numer. Math. 103 (2006), 667-689.

[33] Z. Zhu, B. Song and J.K. White, Algorithms in FastImp: A fast and wide-band impedance extraction program for complicated 3-D geometries. IEEE Trans. Computer-aided Design of Integrated Circuits and Systems 24 (2005), 981-998. 